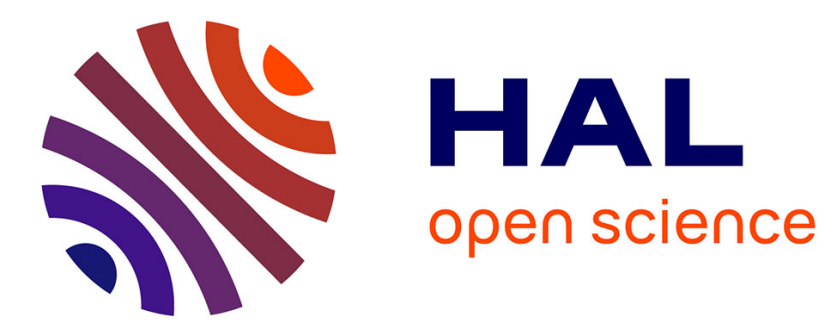

\title{
Les lames très minces et leurs propriétés physiques \\ H.E. Devaux
}

\section{- To cite this version:}

H.E. Devaux. Les lames très minces et leurs propriétés physiques. J. Phys. Radium, 1931, 2 (8), pp.237-272. 10.1051/jphysrad:0193100208023700 . jpa-00233066

\section{HAL Id: jpa-00233066 https://hal.science/jpa-00233066}

Submitted on 1 Jan 1931

HAL is a multi-disciplinary open access archive for the deposit and dissemination of scientific research documents, whether they are published or not. The documents may come from teaching and research institutions in France or abroad, or from public or private research centers.
L'archive ouverte pluridisciplinaire HAL, est destinée au dépôt et à la diffusion de documents scientifiques de niveau recherche, publiés ou non, émanant des établissements d'enseignement et de recherche français ou étrangers, des laboratoires publics ou privés. 


\title{
LE JOURMAL DE PHYSIQLE
}

\section{LE RAIJIUM}

\section{LES LAMES TRĖS MINCES ET LEURS PROPRIÉTÉS PHYSIQUES}

\author{
Par H. E. DEVAUX.
}

D'après la conférence de Pentecòte faite à la Sorbonne

- sous les auspices de la Société française de Physique, le 5 juin 1930.

Sommaire. - La question des couches superficielles des corps et des lames minces est une de celles dont l'importance apparait de plus $\boldsymbol{e n}$ plus clairement aux yeux des physiciens, elle devient une branche nouvelle do la physique. J'ai cherché à donner ici d'abord (chap. I) un exposé historique de la decouverte des lames monomolécu'aires, et à montrer que cette découverte a été faite par lord Rayleigh de 1890 à 1899 dans un cas très particulier, celui des huiles. J'ai eu moi-même ensuite (1903) le privilège de généraliser cette belle découverte en montrant que les subsıances solides peuvent se montrer également sous l'épaisseur d'une seule molécule. Cette extension a permis, dès le début, de mesurer et de préciser, d'une manière purement expérimentale, les dimensions théoriques des molécules. Les coüncidences multiples trouvées, dès le début, entre les val urs théoriques et expérimentales, ont été fr appantes; elles ont été confirmées par les recherches faites ensuite par de nombreux savants. Nais une autre conséquence de ces faits non moins fiappante, et que j'ai soulignée dès 1904, est que l'état solide et l'état liquide se maintenant encore pour des lames n'ayant qu'une seule molécule d'épaisseur, les états de la matière (liquidité et solidité) ne nécessitent nullement l'existence d'agrégats moléculaires.

Dans le chapitı e II j'ai exposé surtout les propriétés physıqu s des lames monomoléculaires: leus manifestations optiques, leur stabilite (si remarquable en face de linstabjlité des lames épaisses) et ensuite leurs propriélés mecaniques, que j'ai particulièrement étudiées parce que ce sont les seules propriétés qui se maintiennent encore intactes quand la matière ne se présente plus que sous une seule molécule d'épaisseur. Telles sont la cohesion avec la limitation en volume; l'expansibilité, qui n'existe notable que pour quelques substances et est analogue à la volatilité ou à la solubilité; la fluidité des lames liquides et l'équilibre spécial qui sétablit entre la lame monomoléculaire et les globules liquides en excès; la rigidite des lames soludes avec ses variantes; enfin la perméabilité.

L'étude des rapports de la lame avec son support à permis d'apprécier la puissante adherence qui existe entre les deux, en rapport étroit avec les phénomènes d'adsorption, et de souillures : celles ci sont souvent monomoléculaires, et viennent tellement modifier la mouillabilité qu'on peut affirmër que le champ d'attraction moltculaire est certainement inférieur au diamètre d une seule molécule. L'existence de lames monomoléculaires htmimoullables le prouve complètement, et prouve, du même coup, la polarite des molécules. Jinfin une étude spéciale du frottenient montre la résistance prodigieuse des enduits monomoléculalres, et la nature rurement moléculaire des phénomines de glissement et de grincement.

Avec le chapitre III j'aborde la structure des lames monomoléculaires en me basant sur la théorie de l'orientation des molécules à la surface des liquides, due à Harkins, Langmuir, etc... J'ai pensé que cette orientation donne une explication très claire de l'hémimouillabilité et qu'on pourrait obtenir expérimentalement des surfaces solides à molécules orientées. L'expérience à remarquablement confirmé cet'e prévision, car j'ai pu réalier des surfaces solides hémimouillables, sur des substances très variées, et ceci mème dans le cas des molécules monọatomiques (métavx) ce qui démontre la polarité des atomes.

Le dernier chapitre se rapporte aux lames d'albumine et à la s/ructure moléculaire des êtres vivants, c'est-à-dire aux problèmes les plus fassionnants que l'esprit humain puisse aborder. Je démontre que la structure intime de la cellule va jusqu'à l'orientation de toutes les molécules, le long des surfaces et à la polarisalion des affinités et des activités, de telle sorte que nous apercevons enfin la cause profonde qui lie la vie et l'activité vitale à l'organisation de chaque être vivant.

eb jocral de physique et le radium. - Série vil. - t. II. - No 8. - aOUt 1931. 


\section{Chapitre I}

1. Introduction. - A l'occasion de son exposition annuelle de Pentecôte, la Société française de Physique m'a demandé de venir exposer devant elle et devant ses invités, en une conférence publique, l'ensemble de mes recherches sur les lames très minces et leurs propriétés. Les pages qui suivent représentent cet exposé, remanié en vue de la rédaction. L'étendue du sujet ne m'a pas permis du reste de donner des détails sur l'ensemble, considérable aujourd'hui, des recherches faites par les auteurs, de plus en plus nombreux, qui se sont occupés des lames minces. Je me suis donc attaché à donner un aperçu historique de la question à ses origines et au début de ses développements, en décrivant plus spécialement mes recherches personnelles.

C'est devant la Société de physique, en mars et avril 1904, spécialement à la séance de Pàques, qu'a été démontrée définitivement la réalisation expérimentale des lames monomo. léculaires, liquides et solides $\left({ }^{28}\right),\left({ }^{29}\right),\left({ }^{30}\right)$; c'est devant elle que les principales propriétés de ces lames ont été exposées et montrées à diverses reprises par des expériences publiques.

Il était naturel, dès Iors, que ce fût aussi devant la Société de physique qu'eût lieu un exposé d'ensemble de la totalité de ces recherches de physique moléculaire auxquelles j'ai consacré beaucoup d'efforts mais qui m'ont aussi donné beaucoup de joies. Entre toutes ces joies, je tiens à signaler celle d'avoir constamment trouvé, auprès de mes collègues de la Société, un accueil et des marques répétées d'estime qui ont été pour moi le plus précieux des encouragements. Je les prie tous de trouver ici mes bien sincères remerciements, mais je tiens à mentionner spécialement Messieurs Guillaume, Brillouin et Perrin, les amis de la première heure, dont les avis m'ont été particulièrement précieux.

2. L'extension de l'huile et des vapeurs sur l'eau et sur le mercure et les actions mécaniques qu'elles peuvent produire. - On connaît de temps immémorial la puissante extension des huiles sur l'eau, et chacun a vu les taches multicolores qu'elles produisent à la surface de ce liquide. La périphérie de ces lames est habituellement blanche et de plus en plus pâle, ce qui démontre que leur minceur va bien au delà de celle donnant les teintes d'interférences auxquelles les bulles d'eau de savon doivent leurs jolies couleurs (fig. 1).

Or, malgré leur minceur extrême, ces pellicules grasses ont une action puissante, pouvant même calmer les mouvements des vagues de la mer, fait remarquable observé depuis des siècles et qui fût l'objet de communications spéciales, en 1887, par l'amiral Cloué $\left({ }^{5}\right)$.

Or, vers cette même époque, en $1888\left(^{6}\right)$, j'arrivai de mon côté à établir expérimentalement que les lames très minces sont capables de produire des actions mécaniques très marquées et en particulier un travail continu, par de simples différences de tensions superficielles. C'est alors, en effet, que j'ai imaginé le jouet scientifique représenté figure 2 et au sujet duquel je m'exprimais dans les termes suivants $\left(^{6}\right)$ (Devaux, 1888, p. 331 ):

«C'est un bateau taillé arec des ciseaux dans une mince feuille d'étain, et échar cré en « arrière; jeté sur l'eau, il flotte facílement; avec une pipette je plaçai une goutte d'alcool “ sur l'arrière, à toucher l'eau, et je vis aussitôt le bateau filer brusquement. Il semble vrai« ment, au premier abord, qu'une répulsion subite et puissante a produit lors du contact « de l'aleool avec l'eau. Mais considérons les faits au point de vue des tensions, des trac“ tions que subit le bateau entouré de toutes parts d'une surface liquide. En avant, sur les " côtés, cette surface est d'eau pure et par suite elle est le siège d'une forte tension ; en " arrière, elle est recouverte d'alcool, et eetto cauche, même très mince, rend la tension “ notablement moindré. Dès lors, sollicité par deux effets contraires et inégaux, le bateau ( cède au plus puissant et se trouve entraîné sans cesse vers la surface libre de l'eau. "

J'ai obtenu les mêmes résultats avec d'autres liquides; à la fin, je me suis servi du camphre et j'ai immédiatement constaté que cette substance présente un avantage considérable : c'est que seules les vapeurs s'étendent alors sur l'eau, de sorte qu'un fragment de quelques centigrammes suffit pour donner pendant des heures entïeres un mouvement rapide et régulier (fig. 3). Le bateau à camphre peut même effectuer un travail imporlant. 
Miri il suffit d'une pellicule d'huile extrêmement minee pour ralentir puis arrêter tout

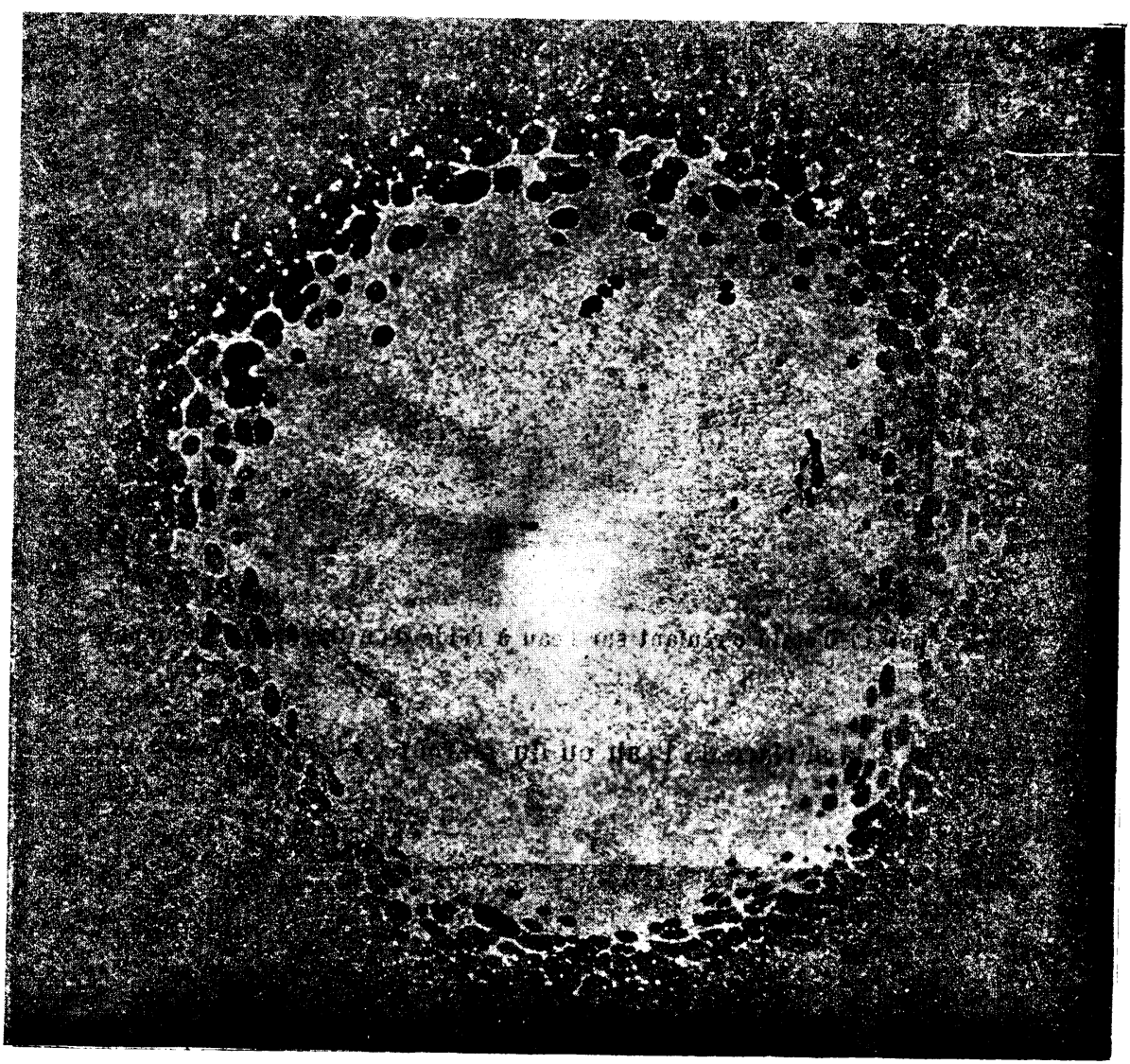

Fiğ. 1. - ( $\left.n^{\circ} 2 \mathrm{y}^{\prime}\right)$. Lame d'huile d’olive, épaisse, à centre blanc entouré d'anneaux colorés, serrés, "mais périphérie mince, ayant la teinte błunche an-dessons du brun de fremier ordre. Celte Jame était agée de 1 seconde quand elhe a été prise. (mor. 1912).

mouvement. L'expérience représen tée I ar la figure 4, Iéalisée plus tard, le prouve directement.

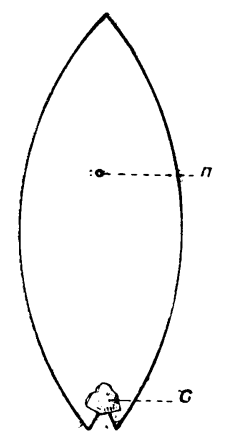

Iig. 2. - Pateau en élain, crandeur naturelle. 
Dès l'année 1888, j'avais donc établi l'êfet puissant de pellicules d'huile ou do

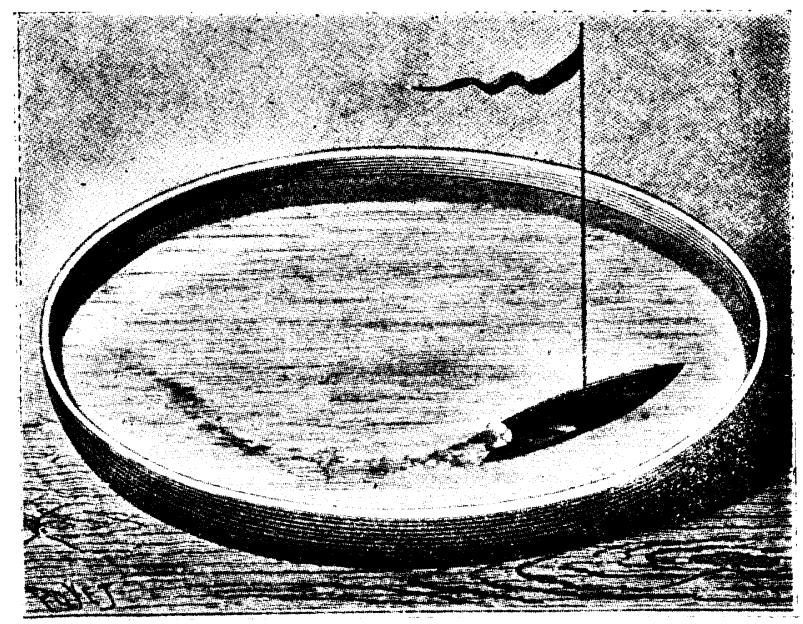

Fir. 3. - Bateau d'étain circulant sur l'eau à l'aide d'un fragment de camphre.

vapeurs de camphre à la surface de l'eau ou du mercure, et j'avais noté l'extrêmo min-

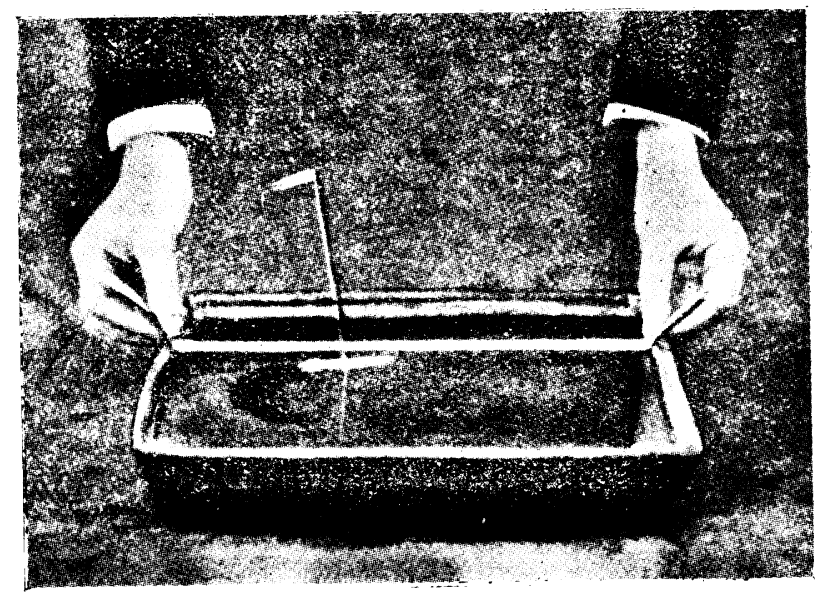

Fig.4. - Bateau en marche dans une cuvette noire, sur de l'eau légèrement huilée, et poudrée avec du tale Un large sillage d'eau camphrée sans talc est produit à l'arrière. L'opérateur rétrécit ou élargit la surface en avançant ou reculant une bande de papier posée en travers. Il rassemble l'huile dens le premier cas, et alors le bateau s'arrète; il l'étend dans le second cas, et alors le bateau repreud sa marche.

ceur de ces pellicules, sans toutefois mesurer leur épaisseur. En réalité, j'avais ainsi réalisé, il y a quarantc-deux ans, un mécanisme mu par l'action d'une lame monomoléculaire. 
3. La découverte d'une épaisseur critique pour les lames d'huile. - Les premières mesures d'épaisseur laites sur les lames d'huile amenèrent à découvrir le fait remarquable que, à partir d'une ceriaine épaisseur critique, la tension superficielle du liquide subit une variation brusque et considérable. Ce fait fondamental a élé établi par les travaux de lorr Rayleigh, de Rionlgeı, d’Agnès Pockel, de Fischer et de moi-même, entro les années 1890 et 1903 .

(1) Lord Rayle gh (1890). - Epaivseur de la couche d'huile suffisante pour arrêter les mouvements du camphre $\left(^{`}\right)$. - C'est lord Rayleigh qui, par des mesures précises, est arrivé le premier, en 1890, à montrer qu'il suffit d'une épaisseur d'huile avoisinant 1 millimicron pour arrèter les mouvements du camphre. Sa méthode est des plus simples. Il pèse une gouttelette d'huile el la dépose sur une large surface d'eau où s’agite du camphre. L'huile s'étend et, lorsqu'il y en a assez, arrète les mourements, ou au moins, les diminue. Par tâtonnements, lord Rayleigh reconnut ainsi :

$1^{\circ}$ que le mouvement du camphre n'est pas modifié tant que l'épaisseur d'huile est inférieure à $1.10^{-7}$ centimètres;

$2^{\circ}$ qu'il est rapidement entravé à partir de 1 et presque complètement arrêté avec $2.10^{-7}$ centimètres.

Le savant physicien attribua cet arrèt à une diminution brusque de la tension superficielle et il émit l'avis qu'alors la lame d'huile est peut-être réduite à 1 ou 2 assises de molécules.'

b) Röntgen (1890). - Sur l'épaisseur de couches d'huile cohérentes, à la surface de l'eau $\left({ }^{9}\right)$. - A l'oceasion du mémoire de loril l'ayleigh, paru en mars 1890, Röntgen décrit,

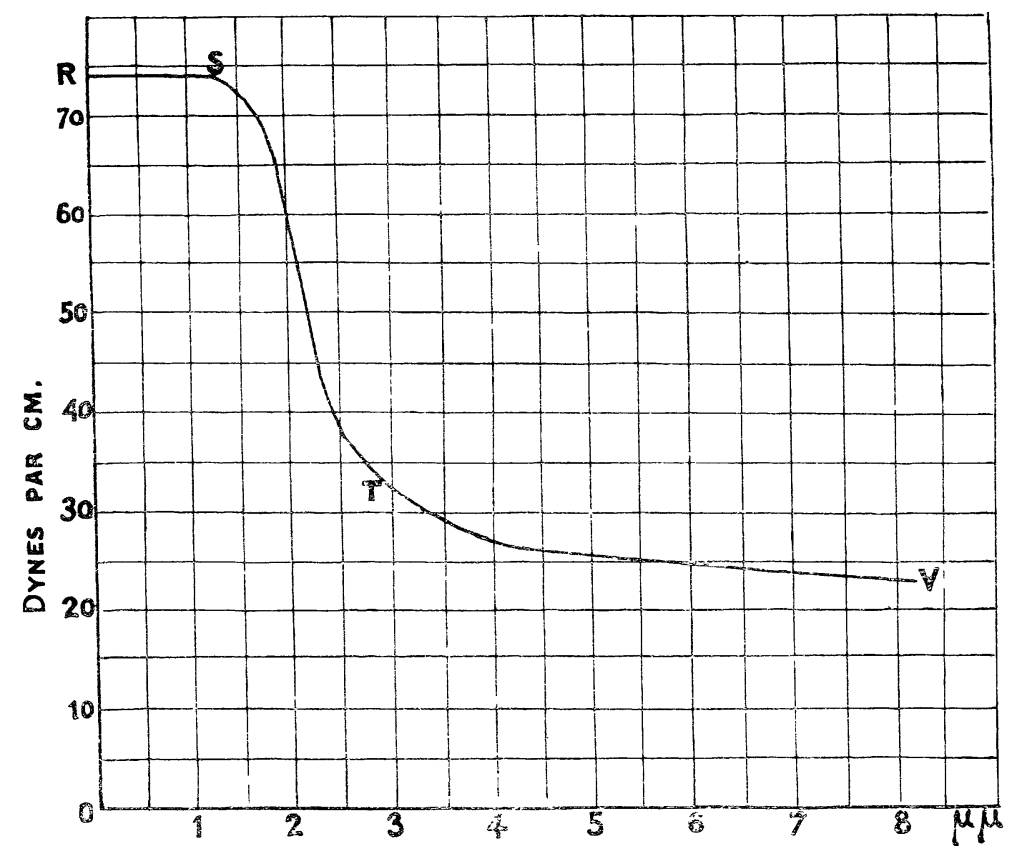

Fig. ら̆. - Courbe, d'après lord Rayleigh, des variations de la tension superficielle de l'eau couverte d'une lame d'huile de ricin, en fonrtion de l'épaisseur de cette lame (supposée uniforme par lord Rayleigh). (Figure empruntée à Langmuir, lor. cit., p. 1859)

en juin de la mème année, une expérience personnelle qui aboutit aussi à démontrer l'existence d'une limite pour l'épaisseur d'une lame d'huileétendue sur l'eau. Des vapeurs d'éther, amenées par un entonnoir, dépriment une surface d'eau, en y abaissant localement la 
tension. Mais si une couche d'huile y est déposée un bourrelet circulaire se forme à une certaine distance de la dépression : le diamètre de ce bourrelet est d'autant plus petit que la quantité de corps gras est plus grande. Röntgen évalue la quantité d'huile minimum nécessaire pour obtenir un bourrelet du plus grand diamètre possible, et admet que, dans ces eonditions, la quantité d'huile a été suffisante pour recouvrir la surface entière de l'eau, d'où il déduit l'épaisseur limite. Il trouva $0,56 \cdot 10^{-7}$ centimètres.

c) Lord Rayleigh et Agnès Pockel (1890 à 1899). - La phase critique de la tension superficielle de l'eau huilée. - Les recherches d'Agnès Pockel, parues en $1891\left({ }^{11}\right)\left({ }^{14}\right)\left({ }^{15}\right)$ $\left({ }^{16}\right)\left({ }^{18}\right)$ confirment l'existence d'une phaśe critique de la tension superficielle des surfaces d'eau huilée, en relations avec l'épaisseur de la couche d'huile. L'auteur qualifie celte phase d'état anormal. Il est à noter que pour faire ces recherches l'auteur a imaginé une technique perfectionnée, en particulier l'emploi de gouttes calibrées d'une solution d'huile dans la benzine pour répandre sur l'eau un poids d'huile rigoureusement déterminé. C'est elle aussi qui a eu la première idée d'employer une auge rectangulaire dont un bord est une barrière mobile, ce qui donne une surface variant à volonté et mesurable avec précision.

Lord Rayleigh adopta cette technique (12.) ${ }^{\left({ }^{13}\right)}$ et en 1899 , il publia un mémoire $\left({ }^{19}\right)$ nouveau dans lequel il donna les valeurs diverses que prend la tension superficielle de l'eau, selon l'épaisseur de l'huile mise à sa surface. Il élablit ainsi qu'au-dessous de $1.10^{-7}$ centimètres, l'huile est inactive, c'est-à-dire n'altère pas sensiblement la tension superficielle. Entre 1 et $2.10^{-7}$ cette tension baisse rapidement, jusqu'à 72 pour 100 de la valeur qu'elle avait sur l'eau pure. Ensuite elle continue à baisser encore, mais plus lentement jusqu'aux épaisseurs de 6 à $7 \cdot 10^{-7}$ centimètres, que l'auteur n'a pas dépassées. La courbe ci-dessus résume ces faits remarquables $\left({ }^{1}\right)$.

Il est à noter que dans l'exposé de ces recherches, Lord Rayleigh paraît admettre que l'étalement complet de l'huile sur la totalité de la surface disponible a lieu dans tous les cas, soit sous une forme active, pour les épaisseurs supérieures à $1.10^{-7}$ (il admet l'existence de lames de 7 à $8.10^{-7}$ ) soit une forme inactive pour les épaisseurs inférieures à $1.10^{-7}$ centimètres.

d) Fischer (1899). - Les épaisseurs les plus petites des pellicules liquides $\left({ }^{17}\right)$. Fischer emploie le mercure comme liquide support, ce qui lui permet l'étude d'autres liquides que les huiles, en particulier l'acide sulfurique et la glycérine. Il trouve que l'épaisseur des membranes de ces liquides uniformément cohérentes à la surface du mercure, doit être inférieure à $3.10^{-7}$ centimf̀tres pour l'huile de navette, à $2.10^{-7}$ pour la glycérine, et à $1.10^{-7}$ pour l'acide sulfurique.

e) Devaux (1903 1912). - Recherches personnelles. - Depuis ma publication de 1888, je pensais toujours aux lames minces, j'avais mème obtenu des résultats nouveaux, mais je n'osais pas les publier, craignant que ma qualité de biologiste ne les fit repousser par les physiciens. Cependant, en novembre $1903\left({ }^{23}\right)$, une découverte faite sur l'albumine et qui rattachait ces recherches à la physiologie, me porta à commencer à les faire connaître aux physiciens $\left({ }^{26}\right)$.

J'apportai les faits nouveaux suivants :

10 Existence d'une limite à l'extension des huiles neutres sur l'eau (extension maxima).

$2^{\prime}$ Apparition d'une cohésion caractéristique dans les lames d'huile ainsi étendues, dès qu'on les resserre quelque peu, cohésion correspondantà une épaisseur précise que j’appelle épaisseur critique.

$3^{\circ}$ Extension de ces données aux substances solides, par l'obtention de lames minces solides ayant aussi une épaisseur critique, très voisine du reste de celle des lames liquides.

Ces trois ordres de faits sont tous compris dans ma deuxième note de $1903\left({ }^{26}\right)$ dont je crois devoir republier ici une grande partie jarce que, parue dans un recueil peu répandu (Proc. Verb. de la Soc. des Sciences physiques et naturelles de Bordeaux), elle a passé inaperçue.

(1) Courbe empruntée au mémoice où Langmuir (1917) rend compte des travaux de lord Rayleigh et établie d'après les chiffres d’un tableau de cet auteur. 
$1^{\circ}$ L'extension de l'huile sur l'eau est limitée. - (Devaux, déc. 190.3). - a) « Si " l'on touche une surface d'eau libre, poudrée avec du talc, avec un fil capillaire portant une “ trace d'huile, l'huile s'étend sur l'eau suivant un cercle manifesté par l'écartement du

" talc. L'écartement est très brusque parce que la tension superficielle normale de l'eau

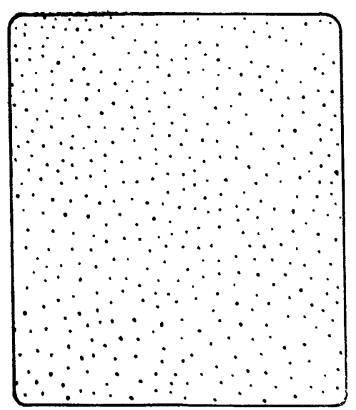

Fig. 6. - Surface d'eau essuyée et légèrement talquée : les grains de poudre de talc sont éparpillés sur toute la surface.

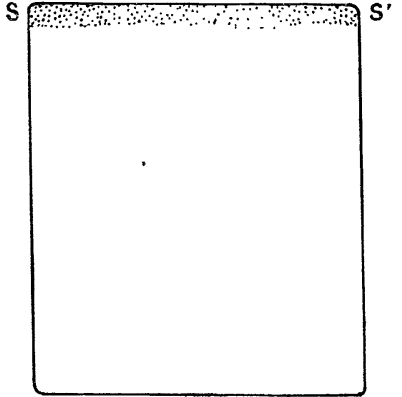

Fig. 7. - Mème surface, sur laquelle on a légèrement soufflé horizontalement; les grains de talc, chassés par le souffle, se sont rassemblés à l'autre bout de la cuvette et intimement juxtaposés; il n'y avait donc entre ex aucune impureté.

« subit un abaissement subit et considérable. Mais le cercle formé est limité; son extension " s'arrête brusquement quoiqu'il subsiste de l'eau libre tout autour. Toutefois, cette eau " est-elle vraiment libre? Des traces d'impuretés pourraient l'occuper et arrêter l'extension « de l'huile. Il n'en est rien. Touchons en effet un autre point de la surface, loin du premier,

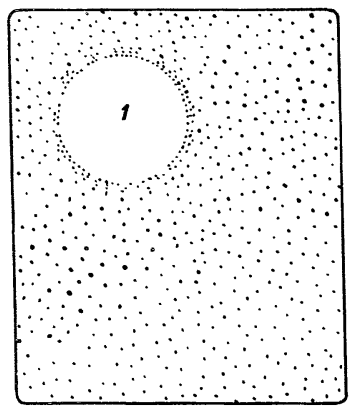

Fig 8. - L'extension de l'huile est limitée. - Surface d'eau légèrement talquée comme dans la figuse e. On la touche en un poiut avec un fil de verre très íin portant une trace d'huile : un cerele très brusque se produit, à extension ne!tement limitée, entouré d'une bordure de talc serrée, tandis qu'au delà les grains de talc sont restés épars, sans aucun déplacement.

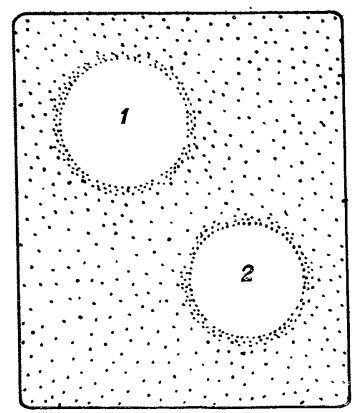

Fig. 9. - Nême surface portant la tache d'huile $n^{\circ} 1$, en extension maxima. Je touche cette surface en un autre endroit ot j'obtiens un deuxième cercle très brusque. Le premier n'est pas modifié. Il n'y avait donc aucune impurelé entre les deux.

« avec une trace d'huile : un nouveau cercle se forme, par écartement du talc, mais le pre-

“ mier cercle n'en est pas modifié ; aucune impureté équilibrante n'était donc en dehors de

« ce cercle; s'il en eût existé, sa surface aurait dû se déformer et diminuer.

"Il y a donc une limite réelle à l'extension de l'huile sur l'eau, et quand cette limite " est atteinte, la tension superficielle est celle de l'eau pure, même pour l'eau huilée ». 
b) “ On peut aussi opérer autrement, répandre d'abord sur l'eau un voile général " d'huile et le poudrer, puis essayer d'en élargir une petite portion. Tout d'abord, la “ surface entière s'étend parce que la couche d'huile est assez épaisse. Mais il arrive tou-

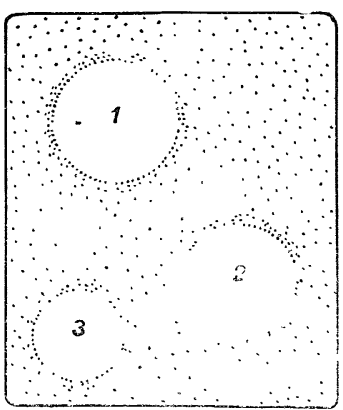

Fig. 10. - Mème surface. Un troisième cercle a été formé loin de 1 el 2 ; le résultat est le mème.

“ jours un moment où l'extension s'arrête: la région huilée, marquée par le talc, reste en " arrière, tandis qu'il se produit une surface d'eau libre, sans talc ni huile. La limite est " très nette, et l'on a côte à côle les deux surfaces ayant une mème tension superficielle: " l'une d'eau libre, l'autre d'eau huilée en extension maxima ».

Examen de la limitation. - Les figures ci-contre (inédites) détaillent tous ces faits. Le fait de la limitation est important en lui-mème, car c'est un caractère de la matière, sous les états liquide et solıde, d'ètre limitée. Elle possède alors un rolume fini el une surface libre si elle est isolée Il en serait de mème ici; une lame mince d'huile possèderait aussi un volume fini et une épaisseur finie; son volume serait représenté par le produit de sa surface générale par son épaisseur.

$2^{\circ}$ Apparition d'ure cohésion par resserrement. - En extension maxin a, une lame d'huile est distendue par l'attraction de l'eau et ne présente qu'une cohésion très faible;

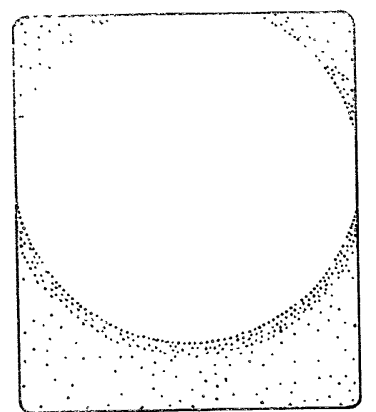

Fig .11. - Ln grand cercle d'huile en extension maxima formé au milieu d’une surface d'eau très lígèrement talquée.

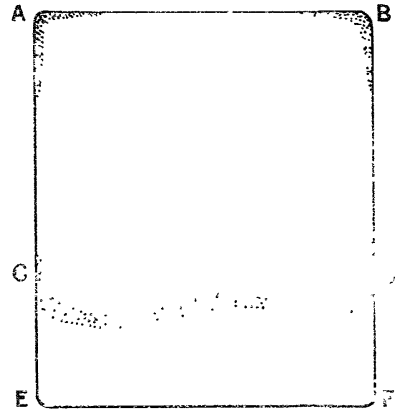

Fig 12. - En souflant doucement le voile d'huile est rassembló à un bout de la cuvette. Le talc est aussi rassemblé dans les deux coins $\mathbf{A}$ et $\mathbf{B}$, et, $\boldsymbol{\epsilon} \mathbf{n}$ travers, selon une ligne irrégulière CD En ECDF, la surface d'eau est rigoureusement pure.

non seulement elle se déforme, mais elle se rompt avec la plus gı ande facililé. Par exemple, si l'on souffle verlicalement sur elle, après l'avoir légè rement lalquée, elle s'ouvre en un çrcle représenté par de l'eau pure.

Une lame d'huile resserrée par ‘une barriè:e mobləs, se condıit d'une toute autre 
manière ; elle possèdc une cohésion puissante de toutes ses parties, aucun souffle n'arrive à la briser. Ln courant d'air vertical n'y produit jamais un cercle d'eau libre, il provoque seulement de rapides courants de convection, centrifuges et centripètes, témoignant que toutes les parties du roile sont solidaires les unes des autres; il en est de même d'un courant oblique ou horizontal et de toutes les tentatives de modifier l'étendue du voile, soit par extension, soit par compression. Les moindres variations forcées, en un point quelconque, se transmettent à l'ensemble. C'est ainsi, par exemple, que, lorsqu'on rapproche doucement la barrière, au moment précis où le talc situé en avant du voile manifeste un resserrement, les petites taches de talc, situées partout le long des bords, même les plus

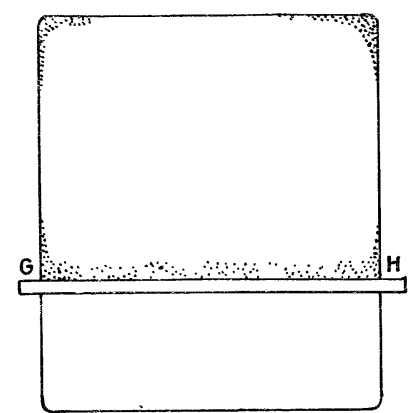

Fig. 13. - On rectifie la limite du voile d'huile par une barrière mobile GH.

éloignées, subissent le mème resserrement; le voile huileux tout entier se conduit comme un bloc qu'on serait tenté de qualifier de rigide si, d'autre part, la fluidité n'était pas des plus manifestes.

En un mot, sous la forme de lames très minces étendues sur l'eau, mais un peu resserrées, lhuile a un volume précis et une cohésion considérable, comme lorsqu'elle est en masse.

Ces conditions sont éminemment favorables pour des mesures d'épaisseur, il suffit de mettre sur l'eau un volume connu de l'huile et de mesurer la surface qu'il prend lorsqu'il est étendu en voile légèrement serré. Le quotient du volume par la surface donnera l'épaisseur.

3‘ Mesures d'épaisseur. - C'est cette épaisseur que j'ai appelée au début épaisseur critique, ne voulant pas préjuger de sa nature

Pour faire ces mesures, j'employais au début de simples cuvettes à photographie, que je remplissais d'eau ; sur cette eau, je déposai 1 ou 2 gouttes calibrées d'une solution d'huile dans la benzine. Ces gouttes s'étendent, puis s'évaporent, laissant un léger voile sur l'eau. J'employais ainsi, sans le savoir, la méthorle imaginée par Agnès Pockel dès $1891\left({ }^{(1)}\left({ }^{14}\right)\right.$ et adoptée par lord Rayleich, mais c'est le seul point de ressemblance de nos recherches, car au lieu de porter mon attention sur les variations de la tension superficielle, je l'appliquai à examiner celles de la cohésion de la lame. Donnons un exemple d'après mes recherches ultérieures [Devaux 1912, $\left({ }^{37}\right)\left({ }^{38}\right)$ ].

"Je prépare une solution titrée d'huile dans la benzine pure. Je me suis servi, en " dernier lieu, d'une solution contenant exactement 1 centimètre cube d'oléine pure (trio“ léate de glycérine) pour 1000 centimètres cubes et d'une pipette donnanl 50 gouttes de " cette solution pour 1 centimètre cube. Une gontte contient donc 1/30000 de centimètre “ cube d'huile et je dépose deux de ces gouttes sur l'eau.

"Aussitôt tombées, ces gouttes s'étendent sur la totalité de la surface de l'cau, et " l'évaporation de la benzine est quasi instantanée, laissant un résidu d'oléine égal à “ 2/000.000 ou 400.10-7 centimètres cubes. Des mesures antérieures m'ayant lémon/ré « que cette quantité d'huile est incapable de couvrir la totalité de la surface disponible de 
" la cuvette $\left(62 \mathrm{~s} \mathrm{~cm}^{2}\right)$, je souffle de loin pour rassembler le voile invisible d'huile à "l'extrémité élcignée de la cu vette, et je répands aussitôt sur l'extrémité la plus rapprochée " un léger voile de poudre avec le tamis. Le talc tombe ainsi sur l'eau libre; il

a fuit, emporté par le souffle, mais on le voit brusquement arrêté le long d'une barrière

" TT' invisible et des plus nettes, constituée par une lame d'huile $\mathrm{H}$ (fig. 14). L'arrèt est d une «" netteté saisissante.

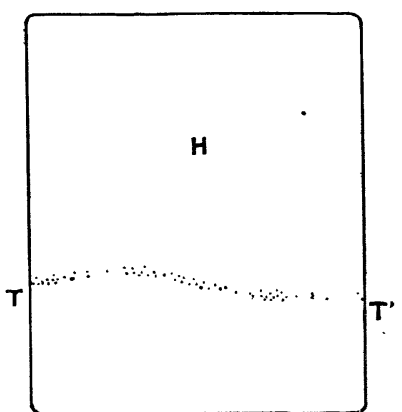

Fig. 14. - Rassemblement de la lame d'huile.

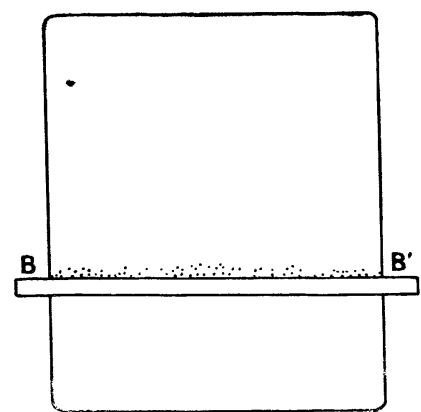

Fig. 15. - Etablissement de la surface par une barrière mobile.

" On applique alors, sur la partie de l'eau sans huile, une bande de papier BB' (fig. 15̃) « pour faire une barrière capillaire à bord rectiligne. Cette barrière e rapprochée douce" ment de la bande de talc, qu'elle redresse, comme on le voit sur la figure 1马. Si alors on " rapproche un peu plus la barrière, on voit les grains de talc, situés juste à la limite de " l'huile, et plus ou moins distants les uns des autres parce qu'ils sont légèrement huilés, " subir un resserrement brusque entre l'huile et le papier. On recule la barrière, et l'on “ voit tout à coup ces mêmes grains redevenir libres, flottant côte à côte sans résistance. «Par de petits mouvements brusques en avant ou en arrière, j'arrive ainsi à préciser, à " quelques millimètres près, la limite à laquelle le voile d'huile est un peu resserré, à " peine, c'est-à-dire au début du changement de tension.

« C'est à cette limite que je prends une mesure, en déterminant au moyen d'un double " décimètre, la longueur de la nappe d'huile; sa largeur est déterminée une fois pour " toutes ».

Résultats. - On a ainsi la surface moyenne occupée par une lame. Cette surface était, dans les expériences faites le 18 avril 1912 de $363,71 \mathrm{~cm}^{2}$. Or, cette surface d'huile a été produite par deux gouttes de la solution, c'est-à-dire par $400 \times 10^{-7} \mathrm{~cm}^{i}$ d'huile. L'épais. seur de la lame était donc de :

$$
e=\frac{V}{S}=\frac{400.10^{7}}{363,71}=1,10.10^{-7} \mathrm{~cm}
$$

avec une approximation allant de 1,04 à $1,15.10^{-7}$. On peut donc dire que "la plus mince lame d'huile cohérente qui peut exister sur l'eau possède 1,1 millionième de millimètre. 》)

๖. Résumé. - Cette valeur est remarquablement voisine de celles trøuvées par les auteurs en se servant de la variation de la tension superficielle :

lord Rayleigh ( $\left.{ }^{7}\right)$ (mouvements du eamphre) ........ 1 à $7.10^{-7} \mathrm{~cm}$

Röntgen $\left({ }^{9}\right)$ (vapeurs d'éther)................

Agnès Pockel (tension superficielle) $\ldots \ldots \ldots \ldots \ldots \ldots \ldots$ ?

lord Rayleigh $\left({ }^{19}\right)$ (tension superficielle)........... 1 à $2-$

Fischer $\left({ }^{17}\right)$ (huile de navette sur le mercure) ......... 3,00 -

H. Devaux $\left({ }^{(38}\right)$ (apparition de la cohésion)........... 
Cette méthode de mesure de l'épaisseur des lames minces, que j’ai inaugurée en 1903, est la seule applicable aux solides comme aux liquides.

Les lames minces solides. - Jai eu le privilège de réaliser le premier des lames solides flottantes, extrêmement minces et, par là, de donner aux observations faites sur les huiles une portée beaucoup plus générale.

a) Dans leurs belles recherches, lord Raỵleigh et Agnès Pockel se trouvaient très limités : ils ne pouvaient étudier que des liquides parce que la propriété dont ils examinaient la variation était la tension superficielle. Et même, parmi ces liquires, ils étaient obligés de se restreindre à ceux qui sont à la fois non volatils et insolubles dans l'eau, ce qui empê. chait toute généralisation.

b) Le choix d'un autre caractère, la cohésion, m’a permis, au contraire, de considérer aussi les substances solides. La cohésion est en effet une propriété très marquée dans les solides, plus complètement même que dans les liquides. On pouvait dès lors s'attendre à une variation intéressante de ce caractère par une minceur suffisante.

Voici textuellement, ce que j'ai publié à ce sujet, dans ma noteinitiale (3 décembre 1903) ${ }^{26}$ ) que j'avais intitulée : « Recherches sur les lames très minces, liquides et solides, existence d’un minimum d'épaisseur ».

“En déposant sur l'eau une goutte, pas trop diluée, de cire ou d'acide stéarique dans “ la benzine, cette goutte s'étend, puis s'évapore et laisse une lame mince de ces substances. "Cette lame est solide. Il est intéressant de chercher s'ilexisteaussi pour ces lames minces « une limite d'épaisseur et si l'état solide se conserve jusqu'à la limite.

“ Les essais faits sur quelques corps montrent avec netteté qu'il y a, en effet, une “ limite d'épaisseur, et qu'à cette même limite l'état solide subsiste encore, quoique très « affaibli (cire d'abeilles et cire végétale, mastic, acide margarique, paraffine, acide stéa" rique, sulfure de cuivre). Pour certains corps (colophane) le fait est moins certain, ce qui « tient probablement à des impuretés liquides.

“ Cette persistance d'un état solide très affaibli avait déjà été révélée par les lames ( minces d'albumine et de corps analogues pour une épaisseur voisine de 2 à $10.10^{-7}$ grammes « par centimètre carré. J'ai pris également des mesures de l'épaisseur minima possible « pour les corps suivants;

\begin{tabular}{|c|c|c|}
\hline Colophane. & $2.10^{-7}$ & \\
\hline Paraffine. . & $0, \check{\partial}$ à $1, \check{\partial} \cdot 10^{-7}$ & \\
\hline Acide stéarique. . & 1,7 à $2.10^{-}$ & \\
\hline Sulfure de cuivre. & 2 à $3 \cdot 10^{-7}$ & \\
\hline Cellulose. . . . . . . & 1 à $2.10^{-6}$ & \\
\hline
\end{tabular}

« Les trois premières substances ont donné des lames minces par l'emploi de solutions " dans la benzine. Le sulfure de cuivre a été obtenu par l'action de $\mathrm{H}^{2} \mathrm{~S}$ gazeux sur une « goutte d'une solution titrée de sulfate de cuivre étendue sur verre. La cellulose a été ( employée en solution dans la liqueur de Schweitzer, laquelle s'étend surl'eau. La valeur « mesurée pour cette dernière substance est, du reste, sujette à revision.

« On voit que l'épaisseur limite des lames solides les plus minces est en général voi« sine de 1 à $2.10^{-7}$ grammes par centimètre carré.

( c) Changement de cohésion avec l'épaisseur. - En rétrécissant la surface occupée par une “ lame mince d'acide stéarique ou de cireà la limite, on voit apparaître et monter rapidement « la cohésion caractéristique d'un corps solide. Il suffit de doubler l'épaisseur pour passer « d'un voile quasi liquide à une membrane nettement solide. Inversement, si l'on cherche “ à étendre de nouveau la surface, le voile tend aussi de nouveau à s'étendre, quoique “ moins nettement que dans le cas des liquides. Mais en mème temps sa cohésion décroît “ très vite; puis l'extension du voile s'arrête à peu près à la limite primitive (sauf si l'on “ avait fortement rétréci jusqu'à plisser la lame), avec une cohésion très faible. On peut « ainsi, par de simples variations de la surface, c est-à-dire de l'épaisseur, faire apparaître « ou presque annuler la cohésion propre de la lame solide. Ce phénomène remarquable est 
“ des plus nets; il est d'une allure exactement parallèle aux variations de la tension super « ficielle signalées plus haut pour l'huile sur l'eau.

"Si la solution employée est trop diluée, il peut arriver que la goutte s'étende sur une “ surface trop grande. Dans ce cas, la substance qui reste après évaporation ne présente " absolument aucune cohésion, pas de solidité simplement visqueuse, ni mème de cohésion " liquide comme une lame d'huile au-dessus de l'épaisseur minima (sauf la colophane). " Mais si l'on rétrécit la surface on voit tout-à coup la cohésion solide apparaitre, puis « monter rapidementjusqu'à l'état solide bien caractérisé. Tout se passe, en un mot, comme « si la substance était formée par dcs grains solides séparés, comme ceux même de la poudre " dont on se sert pour garnir la surface. Tant que les grains ne sont pas au contact, la " surface reste parfaitement liquide malgré leur présence. Dèsqu'ils se touchent, elle devient " rigide ».

כ. Ia découverte dis lames monomoléculaires. - Les dernières lignes ci-dessus montrent que, dès la fin de 1903, les lames obtenues m'avaient fait songer à l'état discontinu de la matière. C'est, en effet, très peu après (mars et avril 1904) $\left({ }^{28}\right)\left({ }^{29}\right)\left({ }^{30}\right)$ que j'ai osé affirmer que les lames très minces réalisaient expérimentalement un but qui aurait paru chimérique autrefois, la réduction de la matière, tant solide que liquide, en pellicules n'ayant qu'une seule molécule d'épaisseur. Mais lord Rayleigh y avait songé treize ans auparavant.

a) L'hypothèse de lord Rayleigh. - Dès 1890, lord Rayleigh dans son célèbre mémoire sur la mesure de la quantilé d'huile nécessaire pour arrêter les mouvements d'u camphre sur l'eau $\left({ }^{7}\right)$ dit qu'une telle mesure, quoique difficile, est d'un grand intérêt pour la détermination des grandeurs moléculaires. Ses mesures lui montrent que cette épaisseur est comprise entre 1 et 2 millionièmes de millimètres et peut être estimée, avec quelque pré cision, à $1,6.10^{-7}$ centimètres, mais il croit que cette épaisseur est beaucoup plus petite que le rayon d'activité des forces de cohésion.

En $1899\left({ }^{19}\right)$ il reprend ses expériences et établit la courbe signalée qui montre une brusque variation de la tension superficielle de l'eau huilée pour une certaine épaisseur d'huile. Cette épaisseur pour laquelle la tension superficielle commence à baisser, est de $1,0.10^{-7}$ centimètres. "Maintenant, « dit-il, ceci n'est qu'un multiple modéré du diamètre “ supposé d'une molécule gazeuse et excède peut être à peine ou pas du tout le diamètre à " attribuer à une molécule d'huile ». El il ajoute : "si les molécules se comportent comme " les sphères rigides de la théorie des gaz, aucune force ne pourra être appelée à entrer en “ jeu jusqu'à ce qu'elles soient complètement rassemblées. Selon cette vue, la tension devrait “ rester constante jusqu'à ce qu'un double champ de molécules commence à se former. Elle “ changerait alors subitement et resterait constante à la nouvelle valeur jusqu'à ce qu'un “ second champ fut complet. L'allure de la courbe de tension s'écarte assez notablement de ( cette description, mais peut-ètre pas plus qu'on ne pourrait l'expliquer par l'hétérogé" néité de l'huile grâce à laquelle certaines molécules monteraient plus facilement que " d'autres, ou à cause des mouvements moléculaires, lesquels ne peuvent être entièrement “ ignorés. Si nous acceptons cette vue comme réellement vraie, nous concluons que la pie" mière chute de la tension correspond à un champ complet d'une molécule d'épaisseur et " que le diamètre d'une molécule d'huile est environ $1,0.10^{-7} \mathrm{~cm}$. »

Langmuir $\left({ }^{3} 3\right)$, citant ce passage, en conclut que lord Rayleigh croit que le premier point d'inflexion de la courbe correspond à un champ d'une molécule de profondeur et que la seconde inflexion correspond à un double champ de molécules.

Quoi qu'il en soit, il est positif que lord Rayleigh a vraiment pensé avoir probablement réalisé des lames monomoléculaires d'huile et nous devons lui rapporter tout le mérite de cette belle découverte.

Quelques années plus tard, en 1903, lord Rayleigh emploie la comparaison suggestive suivante $\left({ }^{23}\right)$ : “ Nous pouvons, dit-il, supposer que 1 millionième de millimètre est le dia" mètre approximatif d'une molécule d'huile; à partir du point où la tension superficielle " est brusquement altérée, il n'y avait qu'une couche simple de molécules sur l'eau. Une “ comparaison fera comprendre pourquoi la tension superficielle était altérée par une “ plus grande quantité. Si une petite quantité de billes flottaient éparses sur du mercure, 
“ elles n'offriraient aucune résistance particulière, si quelqu'un les poussait ensemble vers « un côté du vase; mais il y aurait une résistance si elles étaient assez nombreuses pour " couvrir la surface du mercure et si, repoussées ensemble, elles devaient monter les unes " sur les autres.")

Il est surprenant, dès lors, que lord Rayleigh, ayant son attention attirée de ce côté n'ait pas cherché à savoir si l'épaisseur de ses lames d'huile était vraiment comparable au diamèlre théorique des molécules des huiles, car des formules, données dès cette époque par les physico-chimistes, permettaient de calculer ce dimètre avec précision pour une molécule chimique quelconque (ex Noernst, Theoretische Chemie, $3^{9}$ édition, 1900, p. 394).

b) Recherches parsonnelles. - C'est en cette même année 1903 que je commençai mes propres publications sur ce sujet [Devaux $\left.\left.{ }^{\left({ }^{2}\right)}{ }^{(26}\right)\right]$. Mais au lieu de restreindre mon étude aux huiles. j'avais étudié aussi des substances solides et j'avais trouvé, non sans un très vif intérêt, qu'elles formaient aussi des lames très minces ayant des épaisseurs très analogues à celles des lames $\backslash$ d'huile, quoique spécifiquement distinctes. Je songeai dès lors à examiner si ces épaisseurs étaient comparables aux diamètres théoriques des molécules. Je me servis, à cet effet, d'une formule publiée par Noernst dans le travail signalé plus haut (Noernst, 1900), c'est alors que jeus la satisfaction de constater non seulement que la comparaison était permise, mais que la ressemblance des grandeurs allait dans plusieurs cas à l'identité, aux limites d'approximation existant des deux côtés.

J'eus aussitôt la notion qu'un grand phénomène naturel se dévoilait à nos yeux. Lord Rayleigh avait eu pleinement raison de penser qu'il avait réalisé des lames d'huile d'une seule molécule d'épaisseur. Mais ce fait, bien loin d’être particulier, existait aussi pour beaucoup d'autres substances, et cela sous les deux états solide et liquide : les lames monomoléculaires étaient définitivement découvertes.

1. Premier tableau d'épaisseurs théoriques et expérimentales. -- C'est à la Société française de Physique, dans la séance du 18 mars 1904, que j'ai eu le privilège de donner la primeur de cette découverte $\left({ }^{28}\right)$. Permettez-moi de transcrire ici le passage de la nole qui s'y rapporte:

« On obtient aussi facilement des lames minces solides que des lames liquides...») " Par l'emploi de solutions de plus en plus diluées, j'ai obtenu des lames encore nettement " solides, qui n'avaient qu'une épaisseur voisine de $\mathbf{1} \mathbf{m} \mu$. Toutefois, ici aussi, il existe une " limite infranchissab́le, c'est-à-dire une dimension critique de l'état solide.

" Il est remarquable que cette dimension soit très voisine de la dimension critique " observée pour les liquides. Le lableau suivant, qui indique l'épaisseur critique pour l'huile " et pour divers solides, est démonstratif à cet égard :

\begin{tabular}{|c|c|c|}
\hline - & $\begin{array}{l}\text { Epaisseur critique } \\
\text { de la lame } \\
\text { mince. }\end{array}$ & $\begin{array}{c}\text { Diamètre } \\
\text { calculé } \\
\text { de la molécule. }\end{array}$ \\
\hline CuS & 0,4 à $0,5 \mathrm{~m} \mu$. & $0,2 \supset \mathrm{m} \mu$ \\
\hline $\mathrm{HgS}$. & 0,2 à $0,3-$ & $0,29-$ \\
\hline $\mathrm{PbS}$. & 0,3 à $0,4-$ & $0,31-$ \\
\hline A.gI. . & 1,2 à $1,8-$ & $0,33-$ \\
\hline Acide stéarique..... . . . & 1,7 à 2 & $0,62-$ \\
\hline Spermaceti (palmitate de cétyle?).. & $1, \stackrel{\text { à }}{1,7}-$ & $0,78-$ \\
\hline Huile d'olive (supposée oléine pure). & 0,9 à 1,3 - & $0,94-$ \\
\hline Albumine. & 3,0 à $8,0-$ & $1,87 \ldots$ \\
\hline Stéarate d'albumine. . & $1,25-$ & $1,15-$ \\
\hline
\end{tabular}

“ La deuxième colonne de ce tableau donne les diamètres des molécules calculés « d'après la formule de Noernst. On voit que les lames minces étudiées ici étaient formées " d'une seule assise de molécules, ou d'un très petit nombre d'assises. La dimension critique " ne serait donc autre chose que le diamètre même de la molécule, au moins pour certains « corps liquides ou solides. Cette coïncidence remarquable entraîne comme corollaire immé- 
" diat que les molécules, dans ces lames minces, sont les molécules elles-mp̂mes et non pas " des agrégats de molécules.

“ La cause de l'état solide, au moins pour les substances étudiées ici, ne résiderait “ donc pas dans la grosseur de la molécule; elle est sans doute d'ordre uniquement ciné" tique."

2. Démonstration publique. - La note suivante, publiée à la date du 14 avril, doit ètre reproduite ici en totalité. Elle avait comme titre : Comparaison de l'épaisseur critique " des lames très minces avec le diamètre théorique de la molécule $\left({ }^{29}\right)$ ).

“ Lors de l'Exposition annuelle de la Société de Physique, les 8 et 9 avril 1904, j’ai eu “ l'honneur de réaliser en public les expériences de physique moléculaire dont j'ai déjà ( entretenu la Société ${ }^{1}{ }^{1}$. Je me suis atlaché spécialement à montrer les faits suivants :

" $1^{\circ}$ Existence d'une limite à l'extension spontanée des liquides sur l'eau;

“ $2^{\circ}$ Existence d'une dimension critique pour les substances susceptibles de se réduire “ en lames très minces, dimension caractérisée par ce fait que les propriétés mécaniques " de la substance (tension superficielle des liquides, rigidité des solides), disparaissent « tout-à-coup complètement dès que la lame devient un peu plus minee;

" $3^{\circ}$ Mesure directe des épaisseurs des lames minces à la dimension critique;

“ $4^{\circ}$ Comparaison des valeurs ainsi obtennes avec les valeurs théoriques attribuées ( aux diamètres des molécules.

“C'est spécialement sur ce dernier point, que je m’étais borné à signaler clans mes " communications, que je désire aujourd'hui donner quelques détails.

" A l'Exposition de la Société de Physique, j'ai montré les deux tableaux que voici “ (p. 251 et 222 ), qui permettaient de faire la comparaison : Le premier de ces tableaux “ donne les valeurs numériques, le deuxième représente un schéma de ces valeurs, per" mettant de faire, d'un coup d'œil, la comparaison cherchée.

“Gràce à cette traduction géométrique des résultats de l'expérience et de ceux du " calcul, la ressemblance entre l'épaisseur réelle des lames minces et celle théorique des “ molécules est rendue très frappante. Et, d'autre part, la petitesse excessive des dimensions observées est rendue manifeste par la comparaison avec d'autres dimensions petites déjà connues : épaisseur des couches de passage et limite extrême de la visibilité au microscope. Au grossissement employé ici, un mierobe de $1 \mu$ dediamètre devrait tre représenté par ane bande de 1 mètre de largeur.

“ Il résulte de l'inspection de ces tableaux que les lames minees, à la limite, ont tou" jours des dimensions très voisines des diamètres moléculaires. Et il est important de " remarquer que ces minceurs extrêmes elles-mêmes ne sont pas identiques. Ellles varient, en effet, avec la nature de la substance, et approximativement dans le mème sens que la grosseur théorique des molécules. C'est ainsi, par exemple, que les sulfures métalliques, à molécules très petites, foumissent les lames les plus minces; les corps gras et analogues, à molécules déjà grosses, donnent des lames beaucoup moins minces; enfin l'albumine, à molécules énormes, fournit les lames les plus épaisses. Pour certaines substances, l'épaiisseur observée est identique à l'épaisseur calculée.

“ La concordance de ces valeurs est surtout remarquable parce qu'elles sont complè“ tement indépendantes. Les unes sont purement théoriques, déduites de la théorie cinétique " (chemin de libre parcours đes molécules gazeuses); les autres sont entièrement expérimentales et ne dépendent d'aucune théorie. Une seule concordance de'cette nature serait déjà importante, mais l'existence de concordances déjà nombreuses, et qu'il sera facile de multiplier, assure à un haut degré le contrôle réciproçue entre la théorie et l'expérience. Ces concordances permettent d'espérer que nous sommes en possession d'ure démonstration objective de l'existence réelle des molécules, et de la mesure approchée de leurs dimensions absolues.

" Remarquons du reste, en terminant, que l'existence d'une dimension critique des

(1) Pnocis-berbaux de la Socièté d's Sci,nces physiques et nuturelles de Bordeaux, 19 novembre et 3 déeembre 1903. 
“ substances s'imposait a priori dans l'hypothèse moléculaire, mais pas du tout dans l'hy" pothèse de la continuité de la matière. Dans le premier cas seul il est évident, en effet, “ que la plus mince lame continue possible sera formée d'une assise simple de molécules, “ toute tentative d'amincissement au delà ne fera qu'écarter los molécules, et celles-ci « perdront presque instantanément leur cohésion réciproque. Cette disparition des pro« priétés mécaniques de la substance pour une minceur suffisante est justement ce que “ l'expérience a montré avec évidence, et l'épaisseur correspondante a été appelè dimen. (" sion critique pour ne rien préjuger sur les causes de la variation observée...

\section{Tablead I. - Valeurs numériques.}

« Diamètres moléculaires et épaisseurs critiques des lames minces.

“ $1^{\circ}$. - D'après Noernst $\left({ }^{1}\right)$ le poids absolu d'une molécule quelconque est :

« $P=8,6 \$ 1.10^{-25}$ gramme.

( On en déduit pour le diamètre de la molécule ( $\|$ étant le poids moléculaire quand ( $H=1$, et $D$ la densité) :

$$
d=9, \mathfrak{5} \cdot 10^{-9} \sqrt[3]{\frac{\bar{M}}{D}} \text { centimètre }
$$

( ou bien, en millionièmes de millimètre,

$$
d=0,09 \stackrel{\sqrt[3]{M}}{\frac{M}{D}} \mathrm{~m} \mu .
$$

\begin{tabular}{|c|c|c|c|c|c|c|c|}
\hline & $M$ & $D$ & $\sqrt[3]{\frac{\bar{M}}{\bar{D}}}$ & $\begin{array}{c}d \\
\text { DIAMÈTRE } \\
\text { ABSOLU } \\
\text { DE LA } \\
\text { MOLÉCCLE }\end{array}$ & $\begin{array}{c}e \\
\text { É PA I S SETR } \\
\text { ABSOLEE } \\
\text { DES LAUES }\end{array}$ & & $\begin{array}{c}\text { CONSISTANGE } \\
\text { DE } \\
\text { LA LAME }\end{array}$ \\
\hline Sulfure de mercure .... & 232 & 7,7 & 3,1 & $0,29 \mathrm{~m} \mu$ & 0,29 à 0,45 & $m \mu$ & Solide \\
\hline Sulfure de plomb... . . & 239 & 7,1 & 3,23 & $0,31-$ & 0,27 à 0,42 & - & - \\
\hline Sulfure de cuivre...... & 95,5 & 5,6 & 2,6 & $0,25-$ & 0,4 à 0,5 & - & - \\
\hline Huile d'olive ............ & $880^{\circ}$ & 0,92 & 0,9 & $0,94-$ & 0,9 à 1,3 & - & Liquide \\
\hline Cire du Japon (tripalmitine). & 822 & 0,98 & 9,4 & $0,89-$ & 1,1 à 1,2 & - & \\
\hline Spermaceti............. & 515 & 0,94 & 8,18 & $0,78-$ & 1,5 & - & Solide \\
\hline Acide stéarique ......... & 284 & 1 & 6,57 & $0,62-$ & 1,7 à 2,0 & - & Sol. visq. \\
\hline Iodure d'argent ...... & 235 & วั, 6 & 3,5 & $0,33-$ & 1,2 à 1,8 & - & \\
\hline Stéarate d'alumine .... & 1,753 & 1 (?) & 12,1 & $1,15-$ & 1,23 & - & \\
\hline Acide oléique. . . . . . & 282 & 0,8 & 7,06 & $0,67-$ & 1,4 à 1,00 & - & Liquide \\
\hline Paraffine............. & $"$ & 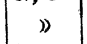 & ) & () & $0, \mathbf{3}$ à 2,3 & - & Solide \\
\hline Albumine .......... & $10,166(?)$ & 1,34 & $19,65(?)$ & $1,8 i(?)$ & 3,0 à 8,0 & - & \\
\hline
\end{tabular}

“ Les valeurs ainsi calculées sont portées dans la כّe colonne du tableau ci-dessous.

“ $2^{\circ}$. - La $6^{\mathrm{e}}$ colonne du mème tableau donne les épaisseurs des lames minces détermi“ nées directement. Deux valeurs y sont indiquées pour chaque substance. La plus faible " indique d'ordinaire la vraie dimension critique, celle pour laquelle la cohésion liquide " ou solide est à sa limite. Commo cette'limite est parfois difficile à préciser avec certitude, “ une deuxième valeur a été donnée, nettement supérieure à l'épaisseur critique. Par “ exemple, pour les suffures $(\mathrm{Hg}, \mathrm{Pb}, \mathrm{Cu}$ ) cette deuxième valeur correspond à l'épaisseur “ que prend la lame rétractée par un excès d'huile mis à côté, sur l'eau qui l'environne. “ Sous cette épaisseur, la rigidité des substanoes solides est considérable. 
Tableau II. - Représentation des la nes très minces.

Epaisseurs limites pour l'état solide et l'état liquide multipliées par un million (I $\mathrm{mm}$ représente $1 \mathrm{~m} \mu$ ).

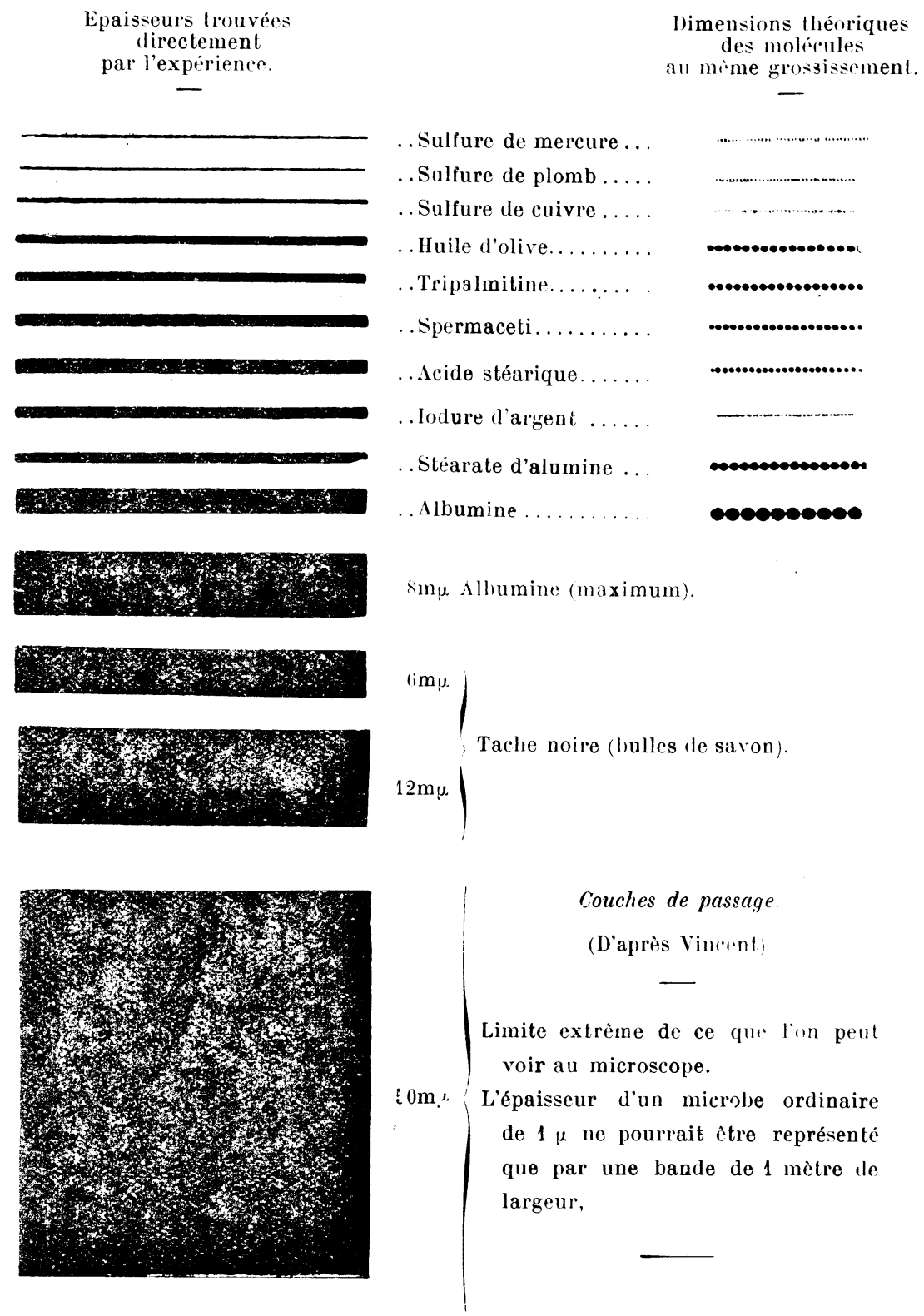

c) Remarque. - L'historique, ici donné, de la découverte des lames monomoléculaires, est fait en tenant comple uniquement des résultats publiés par les auteurs. Pour ce qui me concerne, cet exposé serait notablement différent si je l'avais fait d'après mes notes de laboraloire.

Dès 189', par exemple, j'avais reconnu que, au delà d'une certaine minceur (non 
mesurée à cette époque) la cohésion caractéristique des solides cesse d'exister. Je.croyais،du reste que, à l'état solide, la matière était nécessairement formée de micelles ou du moins.de molécules plus grosses qu'à l'état gazeux et j'y fais allusion dans le sous-titre de ma première communication de 1903. (Essai de détermination de la micelle albuminö̈de). Quant aux dimensions des smolécules, je les croyais voisines de $3.10^{-9}$ centimètres à cause de certains calculs delitippmann ('), de J.-J. Thomson et de moi-mème $\left({ }^{2}\right)$. Je'le croyais encore lorsquejj'eus ohtenu un certain nombre de déterminations expérimentales d'épaisseurs de lames.mimees, liquides et solides. Aussi, lorsque j'eus connaissance, par la formule de Nernst, diune valleur plus précise et moins basse du diamètre théorique des molécules, ce fut avec surprise que je constatai que plusieurs des épaisseurs critiques de mes lames avaient précisément la 'valeur d'un seul diamètre moléculaire. Je trouve encore dans mes notes cette question: " micelles ou molécules?» et même je crus un instant que la micelle de PbS était formée d'une seule molécule! Mais je vis très vite qu'il en était de mème pour le sulfure de Mercure, le-sulfure de Cuivre, et aussi pour le Spermaceti, la Tripalmitine, le Stéarated'Alumine, atc. : toutes ces micelles étaient monomoléculaires! C'était la preuve que, dans ces, cas, la nation dermicelle était inutile et que la particule élémentaire de ces corps solides était la malécule ellc. miême. A fortiori, en est-il de mème pour les corps liquides étudiés : la particule élémentaire des huiles est certainement la molécule elle-mème puisque l'épaisseur expérimentalement trouvée d'une lame d'huile $\left(1,1.10^{-7}\right.$ centimètres) est identique au diamètre théorique de.la molécule de trioléine.

Qn:voit par ces indications que la notion de lames monomolécnlaires s'est imposée à mon asprit malgré des idées préconçues inexactes. Elleest résultée de la vue directe de coïncitlences surprenantes et multiples entre les mesures d'épaisseur des lames et les diamètres théoriques des molécules. Au reste, pour peu qu'on y réfléchisse, la précision de ces coïncidences est stupéfiante puisque l'approximation ne porte que sur les cent millionnièmes de millimètre, et qu'elle est la même, du reste, au point de vue théorique qu'au-point.de vue exprérimental. De telles rencontres ne peuvent ètre fortuites, elles donnent la preuve objective que les molécules on traiment les dimensions calculées et quelles lames monomoléculaires sont des réalités.

C'est ce qu'ont en effet démontré toutes les recherches aultérieures, non seulement les miennes, mais celles aussi des auteurs de plus en plus nombreux qui étudient les lamesitrès minces, en les obtenant non pas sur l'eau seulement mais aussi sur le mercure. Nous dirons un mot ide cess demièras.

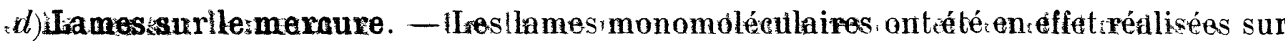
lemereure, comme llet faicaient iprévair les résultats numériques dhtenus parifiseher $\left({ }^{17}\right)$.

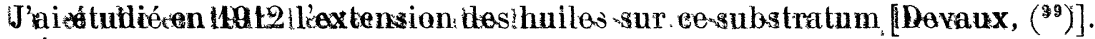

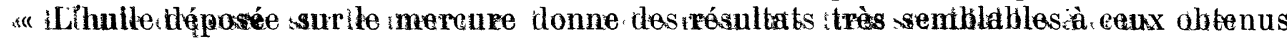

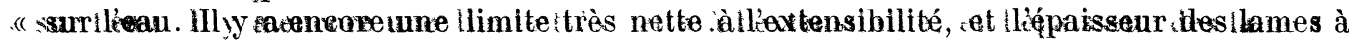

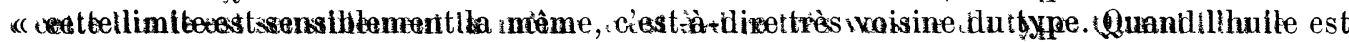
« assez abondante, elle forme ausši une lame épảisse, cólorée, quii évolue rapidement avec ( production de taches noires entourées de perles (fig. 16) et se résout finalement en une “ lame très mince :parsemée de gouttelettes. 'D'autres 'liquides (acide sulfurique, eau de " savon, eau distillée).m’ont donné sur le mercure.des évolutions analogues. Nous sommes ( donc en présence de faits ayant une portéegénérale.»)

D'autres auteurs ont entrepris de'leur côté des recherches analogues, ‘en'particulier J.-J. Haack, $1923\left({ }^{68}\right)$; C.-A. Ressler, $1923\left({ }^{69}\right)$ et R.-S.'Burdon, $1926\left({ }^{79}\right)$. La rapide altération de la surface du mercure entravait toutes les recherches faites avec ce liquide. Mais j'ai réussi, en 1926, à réaliser un nettoyage très rapide de cette surface, par un dispositif très simple [Devaux $\left({ }^{85}\right)$ ] grầce auquè lijai 'pu obtenir sars difficulté avec des substances variées tdes lames très minces. Ces lames se sont montrées encoreidentiques avec les épaisseurs.mono-

(1) Journal.de, physique, $2^{\mathrm{e}}$ série, 2 (1883), p. 113.

(2) Devaux. Rèlation entre'la compresstbilité et la tension superficieltedes liquides (Mém. Soc. sc. phys. et nat.'de'Bordeaux (1894), rp.'3). 
moléculaires (acide abiétique, huiles diverscs, acide sulfurique; glycérine, soufre, ele. Plus récemment. Fahir Emir $\left.{ }^{88}\right)$ se servant du même appareillige que moi, a ob!enu d'autres

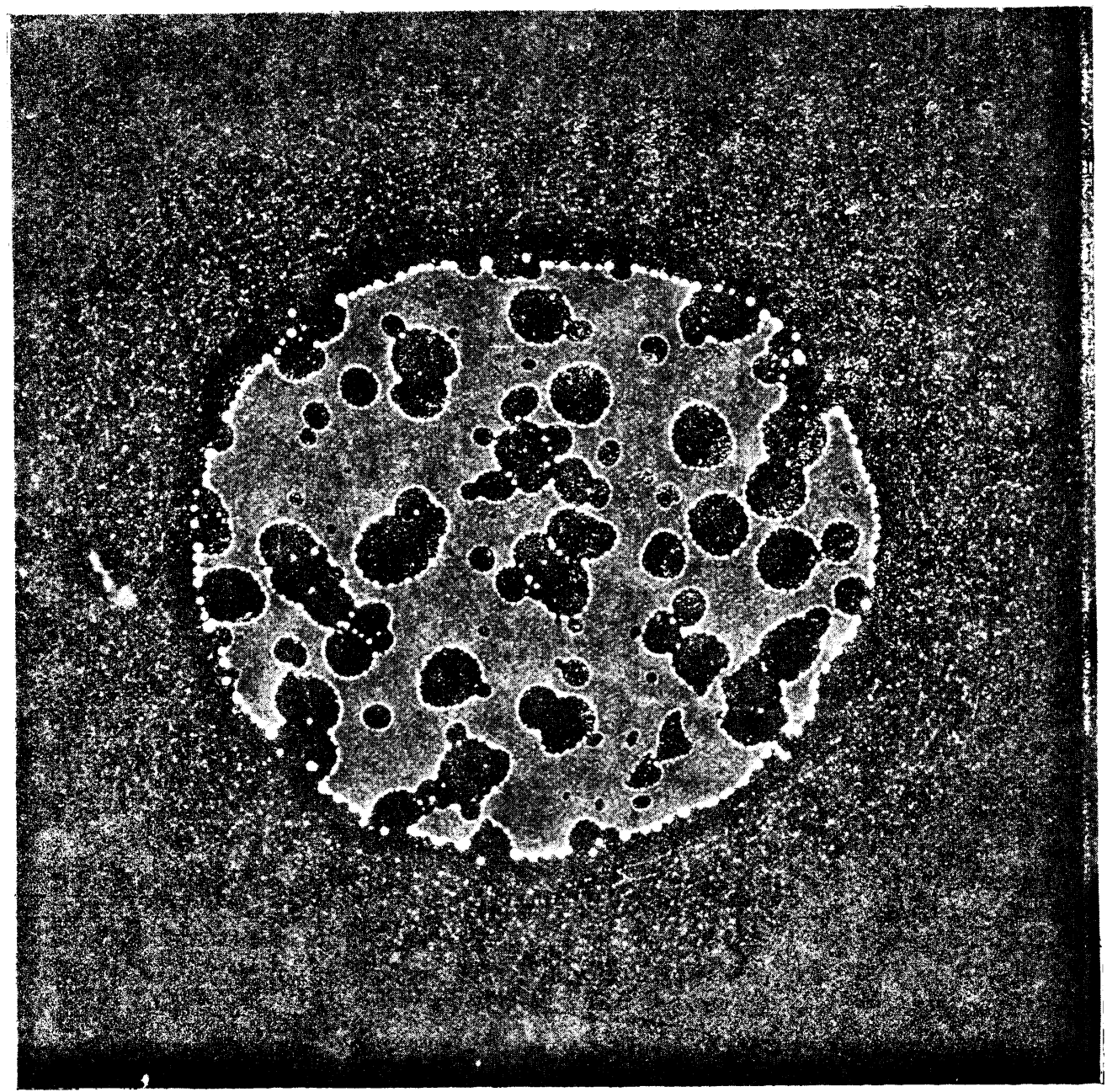

Fig. 16. - Acide oleique sur le mercure. - Lame de teinte chamois $(<100 \mathrm{~m}$ u.). La surface du mercure avait déjà reçu une goutte antérieure qui s'était entièrement résolue en gouttelettes. En conséquence, la goutte nouvelle ne s'est élendue que lentement, en gardant un bord neltement délimité. La rétraction a commencé sur ce bord, par formation d'un bourrelet vite résolu en perles, et abandon d'une zon obscure, sans gouttes, toul autour. Mais il est apparu aussi des taches noires circulaires, à diver: moments, bordées d’un collier de globules, et grandissant progressivement jusqu'à confluence (19 oct. 1912).

mesures analogues, de sorte que le mercure apparait dès maintenant comme un support de choix pourla formation de lames minces monomoléculaires.

e) Conclusion - C'est à lord Rayleigh qu'appartient la conception de lames monomoléculaires et leur réalisation positive dans un cas, celui des huiles. Mes recherches personnelles m ont permis d'achever la démonstration pour les liquides et de l'étendre à diverses substances solides, c'est-à-dire d'établir, comme un fait expérimental, la possibilité de 
réduire lá matière, sous ses deux états, en couches monomoléculaires. Toutes les recherches ultérieures n'ont fait que confirmer cette conclusion en l'étendant à une multitude de subs tances. Nous verrons mème que des divergences trouvées entre l'épaisseur expérimental. des lames et le diamètre théorique des molécules ont pu aboutir à une hypothèse féconde. celle que les molécules ne sont pas isorliamétriques et que de plus, dans les lames monomo. lérulairrs, elles sont orientées.

\section{Chapitre II.}

\section{Les propriétés physiques des lames monomuléculaires.}

La réduction de la matière en lames monomoléculaires étant un phénomène extrèmement fréquent clans la nature, et d'une réalisation très facile pour une multitude croissant.

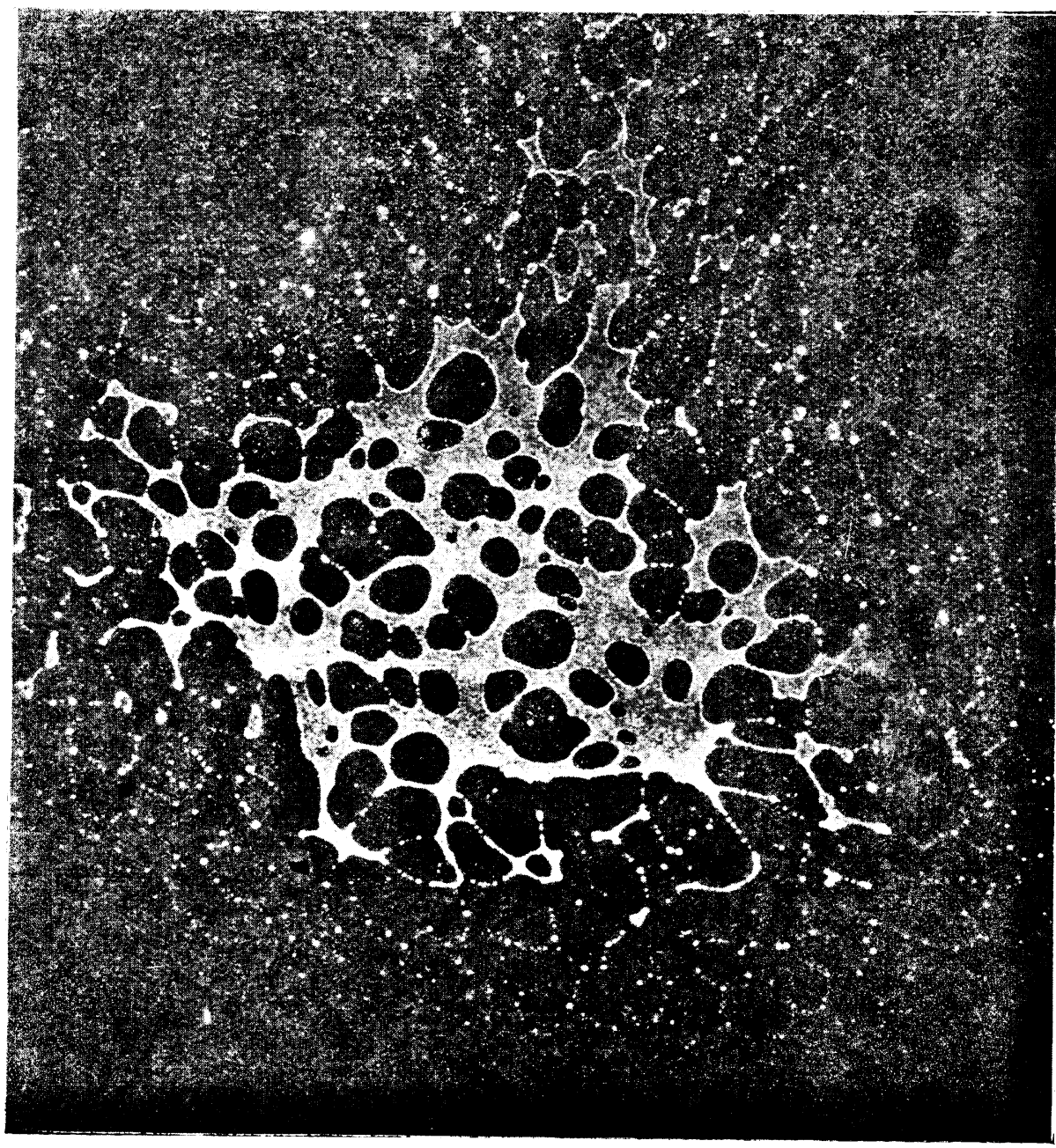

Fig. 17. - Lame d'huile d'olive, vieille de 12 secondes, et saisie en pleine évolution. La période d'étalc ment (teinte pourpre au bord, verte et bleue au centre) a été suivie d'une rétraction générale, déjà terminée à la périphérie (goultes éparses), s'effectuant encore au milieu par l'agrandissement et la confluence des taches noires bordées de perles d'huile (janvier 1913). 
de:substances, létudades propriétés.de da matière sous cette forme remarquable est importante. Nous envigagerons seulement quelques-unes de ces, propriétés.

1. Propriétés eptiques. - La minceur extrème.de ces lames les rend sersiblement invisibles Ill n'yua:guère;que les lames de substances très, réfringentes et très opaques, les sulfures métalliques en particulier, que l'on aperçoit encore, sous.l'apparence d'uno lame très délicate, réfléchissant un peu mieux la lumière que la surface libre de l'eau, etcolorée en brun très påle. Les substances incolores et de faible densité (huiles, graisses, cires, albuminoïdes, etc.) scnt tout à fait invisibles.

Pourtant les impuretés (graisses, etc.) occupant la surface de l'eau se manifestent optiquement en provoquant une polarisations elliptique riégative observée diabord par Jamin ( ${ }^{1}$ ), puis étudiée par lord Rayleigh $\left({ }^{13}\right)$ et dont les manifestations ont été réétudiées en détail 'tout récemment par Charles' Bouhet $\left({ }^{93}\right)$ : Ce dernier auteur confirme en particulier l'orientation des molécutes pour la couche d'impuretés supcrificielles.

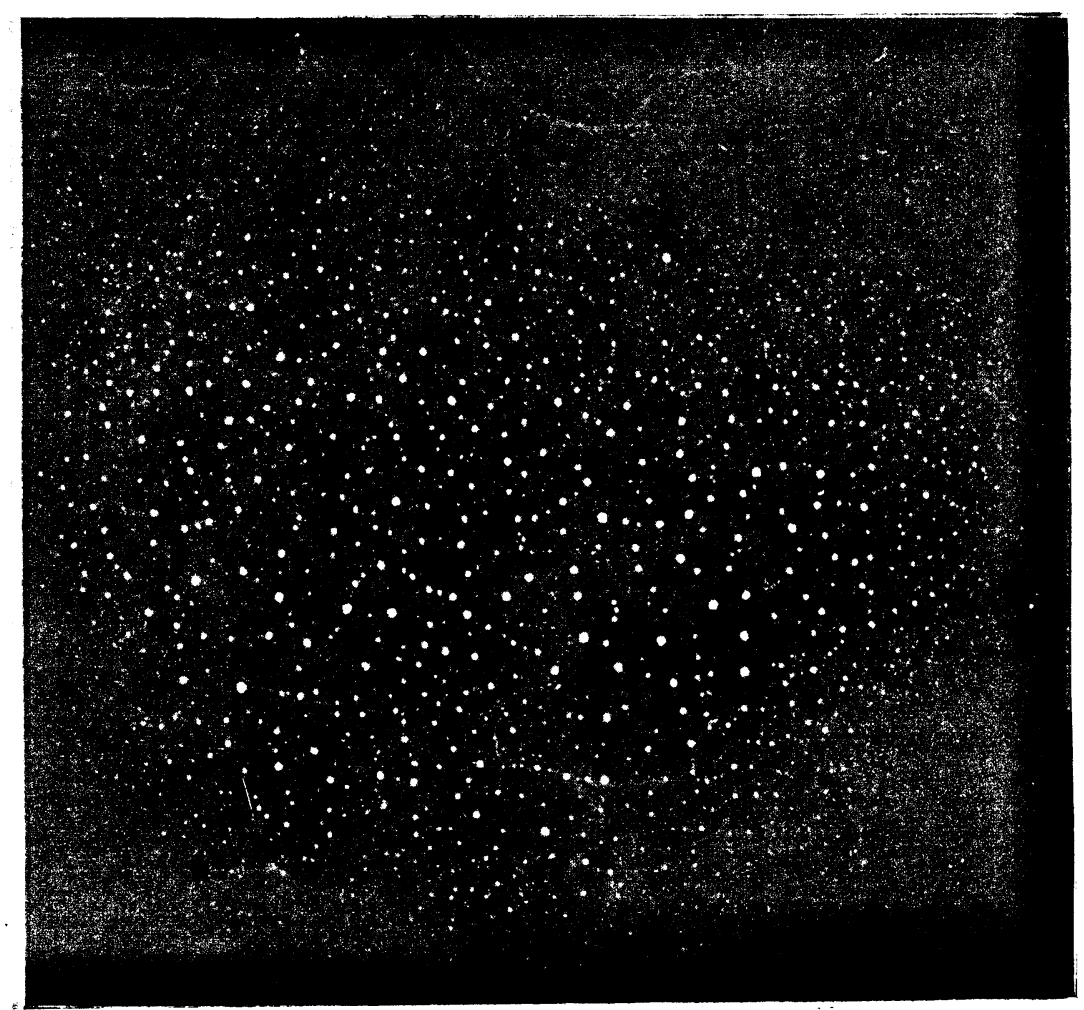

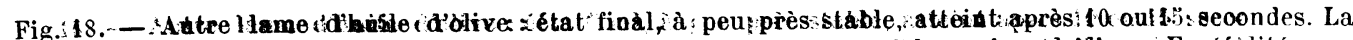

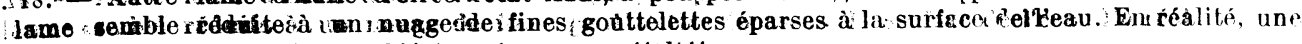

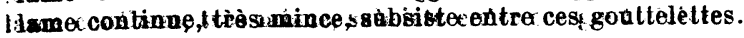

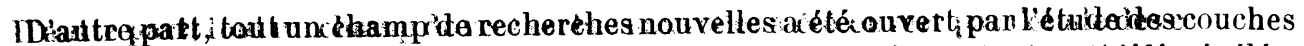

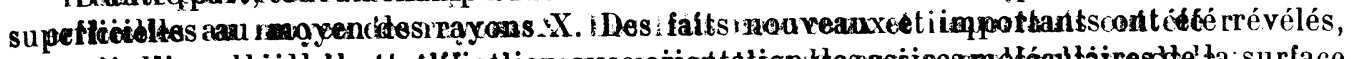

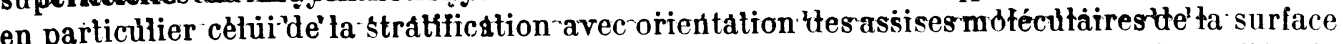
[Thibaud, Trillat $\left.{ }^{(80}\right)\left({ }^{92}\right)$ ]. Ces faits importants sont à rapprocher de ceux fournis par l'étude des' lames 'd'eau'de savon, où'Perrin et ses celèves ont' observé égałement une"structure en feuillets stratifiés [Perín, $\left.\left({ }^{54}\right)\right]$.

2. Stabilité des lames minces, évolution des Lames épaisses:- La stabilité des lames 
monomoléculaires se manifeste spécialement pour les lames monomoléculaires des huiles; elle fait contraste avec l'instabilité de ces mèmes lames lorsqu'elles sont en couches épaisses: Quand une goutte d'huile s'étend ssur l'eau ou sur le mercure, elle donne d'abord une nappe épaisse présentant d'ordinaire les teintes chromatiques (fig. 1). Mais sous cet état la lame est instable, elle se perce très vite en général, de trous circulaires qui grandissent et confluent en un réseau (fig. 17) lequel se résout à son tour en gouttelettes éparses: Mais, entro ces gouttelettes; il reste une lame monomoléculaire, laquelle se montre parfaitement stable et subsiste indéfiniment (fig. 18).

J'ai étudié en détails cette évolution sur l'eau avec diverses sortes-d'huiles [Devaux, $\left({ }^{38}\right)\left({ }^{40}\right)\left({ }^{4.7}\right)\left({ }^{48}\right) \mid$ elle présente toujours ce type, et il en est de même des lames formées sur le: mercure ave les huiles et dautres liquides (Devaux).

L'épaisseur'd'une lame monomoléculaire varie du reste:très peu, comme nous: le ver: rons, quand' om la resserre ou quand on l'étend, par suite d'équilibres spéeiaux qui.słéta: blissent en partieulier avee les globules si elle en possède.

C'est ce que Jontréle tableau ci joint que j'ai publié en 1912:( $\left.{ }^{38}\right)$.

Tablead III. - Représentalion théorique des lames noires et des moléculés.

Minimum et maximum d'épaisseur d'une lame d'huile stable étendue sur"l'eaux:

Epaisseur multipliée par 1 million ( $1 \mathrm{~mm}$ représente $1 \mathrm{~m} \mu$ ).

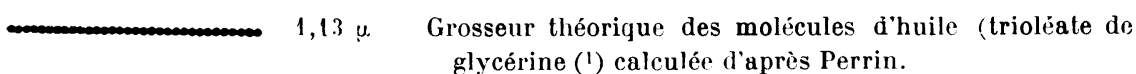
glycérine (1) calculée d’après Perrin.

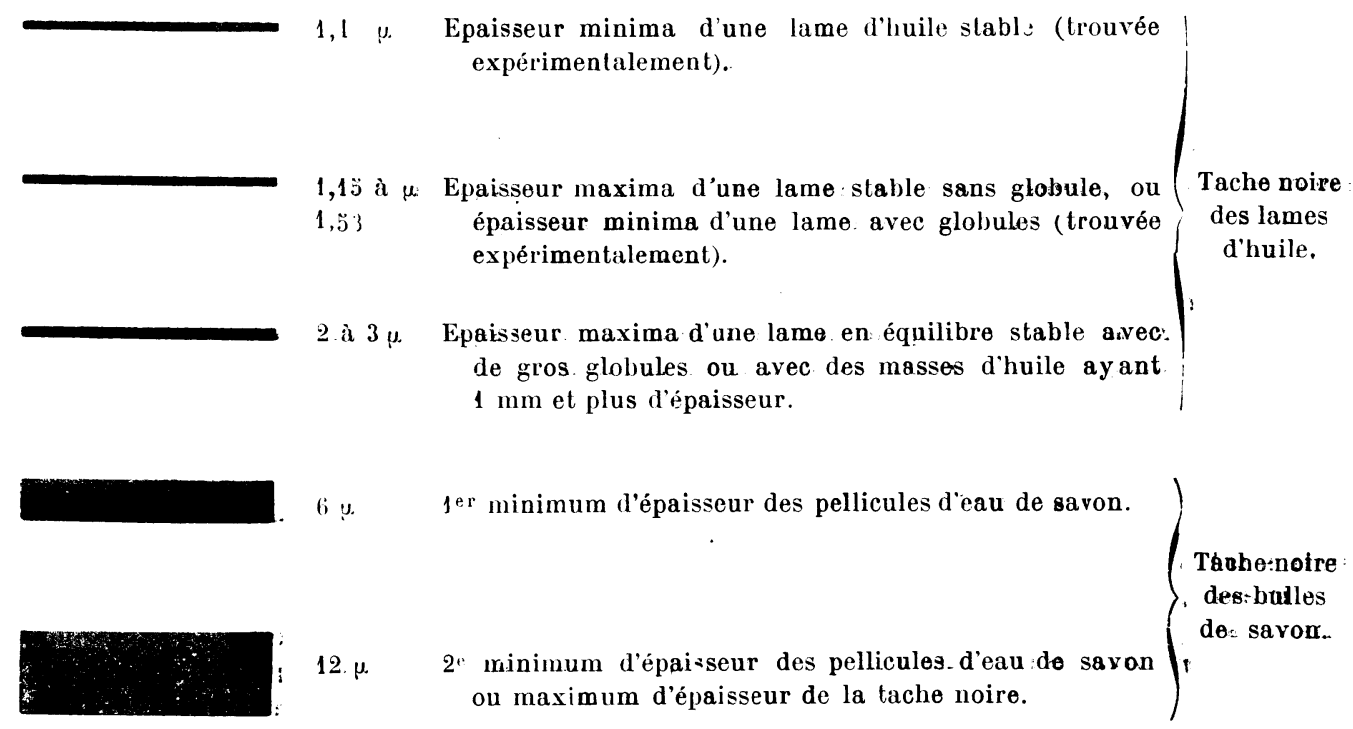

Les causes: de cette stabilité remarquable des lames mono-moléculaires liquides sont aujourd'hui nettement établies gràce surtout aux travaux des physiciens anglais et améri: cains. Elles résident daus les conditions dynamiques. existant le long.des surfaces. Mais ces. mèmes conditions donnent aux lames monomoléculaires des propriétés mécaniques. trèsmarquées, comme nous allons le voir.

3. Proprietés mécaniques. - Si, en effet, les propriétés optiques de lames mon@mor léculaires sont peu manifestes, il n'en est pas de même de leurs propriétés mécaniques. 
C'est certainement par celles-ci qu'on arrive à révéler le plus facilement l'existence même de ces lames à la surface de l'eau ou du mercure. Une substance élrangère, étendue sur ses liquides, tend toujours à donner à cette surface sa tension superficielle propre s'il s'agil d'un liquide, sa rigidité s'il s'agit d’un solide, et ceci méme lorsquell’ est réduite à une seule épaisseur moléculaire. Ce fait remarquable prouve que les états de la matière, caractérisés au point de vue mócanıque (fluidité des liquides et rigidité des solides) résident dans les molécules elles-mémes et ne nécessitent nullement l'existence d'agrégals moléculaires [Devaux $\left({ }^{(\hat{b}}\right), 1904$ ], sans pourtant exclure la possibilité de l'existence de lels agrégats.

$a ;$ G shèsion. - A ce sujet, il e.t important de noter de nouveau le fait, reconnu dès le début de mes recherches [Devaux, $\left({ }^{26}\right)$ ], que la cohésion de lames monomoléculaires se manifeste comme tension superficielle chez les liquides et comme rigidité chez les solides. C'est la preuve directe que ces deux manifestations mécaniques sont dûes à une même cause, l'attraciion moléculaire. Mais certaines lames monomoléculaires sont également susceptibles de manifester une expansibilité ressemblant à celle qui existe dans les gaz, de sorte que les trois états de la matière sont représentés dans les lames très minces.

Passons sommairement en revue ces états.

b) Expansibilité. - Je l'ai découverte, comme on l'a vu plus haut [Devaux, $\left({ }^{26}\right)\left({ }^{28}\right)$ ] sur l'acide oléique et le savon. Pour ces substances, l'extension sur l'eau va bien au-delà de la formalion d'un film monomolé‘ulaire simple, les molécules continuant à s'éloigner, si elles sont entourées d'eau libre, jusqu'à 10 fois la distance normale qu'elles onl dans l'huile. Elles sont alors écartées les unes des autres à peu près comme celles de l'air libre, et réalisent la chimère d'une lame gazeuse monomoléculaire.

( Il semble certain, disais-je en juin 190 ' $\left({ }^{30}\right)$, que certaines substances sont réellement “ douées d’une puissance d'extension superficielle analogue à l'extension d'une vapeur “ dans une enceinte, ou d'une substance dissoute dans un dissolvant ».

Cette conception a été reprise et développée en 1920ّ par A. Marcelin $\left({ }^{74}\right)$ qui a réussi à établir pour les lames d'acide oléique une loi analogue à celle qui régit les gaz: $F S=K T$, mais avec un coefficient $K$ notablement différent. J. Adams est arrivé de son côté à un coefficient semblable à celui des gaz [Adam $\left({ }^{\circ 9}\right), 19.6$ ]. D'après les recherches récentes de J. Guastella ( $\left.{ }^{94}\right)$ le domaine de la vraie loi des gaz $F J^{\circ}=K T$ ne commencerait qu'à partir de $5000 \AA^{2}$ pour la surface occupée par une molécule, c'est-à-dire lorsque les espaces sont d'environ 7 distances moléculaires. Quand les molécules sont plus rapprochées, le coefïicient $K$ est différent comme l'avait établi Marcelin.

c) Fluidité. - La fluidité des lames superficielles se constate facilement en répandant sur elles un léger nuage de talc et soufflant obliquement sur ce nuage avec précaution. Si le voile est fluide, les grains sont emportés même par un courant d’air très léger (fig. 12, p. 241).

Les films liquides partagent ce caractère avec les films pseudo-gazeux. Mais ils en diffèrent par la cohésion, caractère spécial aux états liquide et solide de la matière, gràce auquel celle-ci se présente avec un volume propre qu'on ne peut faire varier qu'entre des limites très étroites, p. 244.

Entre un film d'huile neutre dilaté au maximum et le même contracté jusqu'à l'apparition de globules visibles, les distances moléculaires ne varient pas de 1 à 2 angstroms. [Devaux, $\left({ }^{47}\right),\left({ }^{48}\right)$ ]. S'il s'agit d'un fılm d'acide oléique surétendu, on peut au contraire lui faire subir de fortes contraclions (dépassant 100 angstroms), jusqu'à la phase où, tout à coup, apparaît une résistance puissante, prouvant que les molécules arrivent à se toucher comme dans une huile neutre; très vite après, des globules se forment.

d) Equilibre entre lames et globules. - Remarquons, du reste, que jamais un film monomoléculaire ne présente, côte à côte, sur une même surface, la phase film condensé, à còté de la phase fılm dilaté, dans un équilibre analogue à celui d'un liquide volatile surmonté de sa phase vapeur. De ce côté, la comparabililé n'existe donc pas. Elle existe au contraire, quand on considère l'équilibre spécial d'une lame liquide parsemée de globules, entre ces globules et la lame elle-même. Une telle lame est entièrement comparable à une vapeur rendue saturante par la présence de liquide en excès. Si on la réduit, 
on augmente la masse des globules, si on l'élargit, on la diminue : la tension de la lame ne varie pour ainsi dire pas.

Au reste, la comparaison peut être poussée jusquà l'influence propre du rayon de courbure. On sait que dans une vapeur en équilibre avec des globules, la tension est plus grande avec des petits globules qu'avec des gros. J'ai démontré qu'il en est de mème dans un film d'huile en équilibre avec des globules; sa tension dépend de la grosseur de cenx-ciDans les deux cas, les goultes fines disparaissent, “ mangées » par les yrosses. Ce fait a ćté parfois mis en doute, il est pourtant des plus faciles à constater [Devaux, $\left.\left({ }^{\prime}{ }^{i}\right),\left({ }^{i 8}\right)\right]$.

Notons enfin, parmi ces notions que nous apportent les lames monomoléculaires liquides, combien est petit le rayon des altraclions moléculaires : un éloignement de 1 à $2.10^{-8}$ centi mètres entre les molécules de l'huile, supprime toute attraction moléculaire sensible. C'est là une donnée remarquable qui nous éloigne beaucoup des conceptions admises encore il y a peu de temps, où l'on croyait que le champ d'attraction moléculaire s'étendait par exemple à $25_{0.10^{-8}}$ centimètres et peut-être davantage [Vincent $\left({ }^{2(1)}\right)$ ].

c) Rigidité. - On reconn'aît qu'une lame mince est solide, à ce qu'elle manifeste de la rigidité. Elle résiste à un effort de cisaillement pourvu que celui-ci ne soit pas trop énergique. On constate très facilement la rigidité d'une lame invisible posée sur l'eau, en y répandant une poudre inerte et soufflant sur elle obliquement: les grains de poussière restent en place, ou y reviennent par élasticité, si du moins le souffle n'est pas trol) violént. La chose est rendue très f̣̣appante quand, à còté, existe un fılm liquide sur lequel les grains de poussière sont violemment emportés par le courant d'air, tandis que les borls solides font l'effet d'une surface glacée.

L'état solide des lames varie du reste dans les couches monomoléculaires comme il le fait dans les corps pris en masse, et c'est un fait bien frappant, à ce point de vue, de voir les lames de sulfure de cuivre, de mercure, de plomb, etc., se casser en fragments anguleux, tandis que celles de cire, de suif, de colophane, se montrent molles et même visqueuses; les lames de silicate de soude formées sur le mercure se brisent comme du verre, celles de caoutchouc sont d'une élasticité prodigieuse, et douées en même temps d'une rétractilité spontanée remarquable, etc., etc.

On peut donc dire, saus exagérer, que les modalités de l'état solide se conservent très nettement et même s'accentuent quand il n'y a plus qu'un seul plan de molécules : le silicate de soude, les sulfures, les oxydes, sont fragiles, la colophane est ductible, le caoutchouc, la cellulose sont élastiques. etc.

L'une de ces qualités est la flexibilité, déterminée par l'extrème minceur des lames monomoléculaires. Toutes les lames minces solides, mème les plus fragiles, se plissent quand on les rétrécit de force, au moyen d'une barrière mobile. Elles cèdent à la pression en donnant des plis très fins, mais très visibles, le long de la barrière, qui les comprime de proche en proche. C'est une jolie expérience par laquelle on arrive à montrer, sans poudre de talc, l'existence d'une lame solide superficielle. Toutefois, si cette lame est de la colophane, les plis s'effacent peu à peu, manifesiant ainsi la fluidilé relative de cette substance. Pour les autres substances, les plis sont permanenis, et lorsqu'on recule la barrière, tantôt ils se détachent en lambeaux blancs flottants parce que la lame a été brisće, tantôt ils s'effacent parce que la lame s'étend et s'applique de nouveau sur le plan du liquide.

Perméabilité [Devaux $\left({ }^{58}\right)$ ] - A l'état monomoléculaire, toutes les lames minces sont très perméables à la vapeur d'eau. Mais quand les lames comprennent plusieurs assises de molécules l’imperméabilité apparaît très vite.

Par exemple, quand on amène de l'hy ḋogène sulfuré sur une solution de sulfale de cuivre, il se produit une lame de sulfure de cuivre S'il y a très peu de gaz, cette lame est mince et peu visible; mais si l'action de l'hydrogène sulfuré se prolonge, son épaisseur augmente, comme l'indique l'accroissement de son éclat. Toutefois, cette augmentation est arrêtée bien avant l'apparitien des teintes chromatiques, paree que la lame devient imperméable à l'hydrogène sulfuré. Son épaisseur est facile à déterminer. On étend une goutte d'une solution titrée de sulfate de cuivre sur une surface connue, puis on la sulfure. Par tâtonnements, 
on arrive à déterminer la plus grande quantité de'sulfate'de cuivre'que peut contenir lax goutte pour que tout le cuivre soit transformé en sulfure. Un calcul simple donne:alors. l'épaisseur de la lame obtenue. J'ai trouvé que cette épaisseur est comprise entre $10 \mathrm{~m} \mu$ et $20 \mathrm{~m} \mu$; elle comprend alors plusieurs assises de molécules. Une lame de sulfure de ouirre de 10 à $20 \mathrm{~m}$ u possède une imperméabilité complète et sensiblement indéfinie à-l'égard de l'hydrogène sulfuré (plus de quarante-cinq jours).

Les substances organiques, cire, paraffine, colophane, etc., donnent des résultats analogues. Mème en couche monomoléculaire; une lame de paraffine montre une imperméabi* lité partielle bien manifeste. Il suffit de former, par exemple, une lame monomoléculaire de paraffine à la surface d'une solution de'sulfate de cuivre; ou bien: sur' du mercure, puis'de sulfurer. La pellicule de sulfure métallique qui'se produit est toujours plus mince sous la paraffine, ce qu'on aperçoit très bien quand on a brisè la lame monomoléculaire avant de sulfurer. Lies fragments de lame de paraffine apparaissent en blanc sur un fond sombre. C'est par ce procédé que, dès 1902, j'ai: déeouvert que l'état solide se maintenait encore même sous l'épaisseur de $1 \mathrm{~m} \mu$. mais non au delà. Mais quand on prolonge l'action de $\mathrm{H}^{2} \mathrm{~S}$, on s'aperçoit que peu à peu la paraffine ou la cire sont traversées; de sorte que les lambeaux de lames minces de ces substances qui se montraient blanohâtres au début; apparaissent bientôt plus foncés et plus opaques: que le reste du voile de sulfure.

\section{Rapports de la lame avec son support.}

a) L'adhérence [Devaux, $\left.\left({ }^{66}\right)\left({ }^{77}\right)\left({ }^{89}\right)\right]$. - Une lame superfieielle quelconque est fortement attirée par le liquidé 'qui'la porte, eau ou mercure. Mais: les attractions qu'elle supporte de la part du liquide sont de deux sortes: les unes sont tangentielles; elles s'exercent à la périphérie de la lame si celle-ci est en extension maximum, les autres sont normales à la surface. Ce sont des attractions tout-à-fait distinctes puisque le rayon d'action efficace est notablement plus petit' que le diamètre d'une'seule molécule (p: 259).

Ce sont les attractions tangentielles qui établissent la cohésion propre de la lame, se manifestant comme tension superficielle si la lame est liquide, comme rigidité si elle est solide.

Quant aux attractions normales, elles appliquent fortement: la lame sur son support et se manifestent comme adhérence.

On peut avoir une idée de la puissance de cette adhérence en considérant pour une substance volatile solide télle que le camphre, soit la vitesse avec laquelle elle est arrachée d'un fragment, soit la diminution de volalilité qu'elle subit'quand elle est étendue en lame monomoléculaire. Les essais sommaires, faits sur le mercure en particulier, ont montré que la volatilité du camphre eu lame monomoléculaire, dilatée, est environ 700 fois plus petite que celle du camphre pris en masse $\left(^{\mathbf{8 9}}\right)$. Il en est de même pour d'autres essences ; et:nous trouvons ici l'explieation des odeurs que prennent les vêtements, les murailles, etc., souvent ces odeurs sont inconnues et j'ai montré récemment [Devaux, $\left({ }^{{ }^{9}}\right)$ 1930], qu'une surface de mereure parfaitement propre absorbe dans l'air, libre ou confiné, en cinq ou dix minutes, assez de matières odorantes pour que l'odeur devienne manifeste, soit' par rétrécissement, soit en envoyant une buée avec l'haleine.

Nous sommes ici dans le domaine d'une multitude de phénomènes, en particulier ceux de l'adsorption et ceux des'souillures des surfaces.

b) L'adsorption - On connaît l'importance énorme des phènomènes d'adsorption, mais ce chapitre est tellement étendu aujourd'hui que je dois me borner à le signaler. Je lui ai consacré des publications diverses, en particulier, en collaboration avec M. Aubel, pour l'adsorption des ions: par le verre $\left({ }^{95}\right)$. Cette adsorption ne porte que sur les-cathions, elle donne instantanément une couche monomoléculaire; mais il y a ensuite pénétration ralentie dans le verre.

c) Les souillu res monomoléculaires [Devaux, $\left.\left({ }^{66}\right)\right]$. - La surface libre de tous les corps liquides ou solides est très habituellement couverte de souillirres. Áucun contact' ne s'étáblit sans lèr intermédiaire et les physiciens sont obligés de s'en préoccuper sans cesse, 
dans toutes leurs.reeherches, søit pour les écarter, seit pour tenir. compte de leur. action. propre:

Du reste; la nature de cessouillures est certainement variée, de même queleur. origine, mais peut-être pas autant qu'on serait porté à.le penser; elle est inconnue encore dans.la plupart des cas, ou seulement présumée (séerétions cutanées, fermentations, , etc.) et il en est:de même de l'épaisseur minima sous laquelle ces souillures arrivent à modifier telle. ou telle propriété phrysique; la mouillabilité en particulier.

J'ai signalé plus haut comment ces sevillures superficielles. viennent modifier la réflexion de laxlumière (ps.256) et:la-volatilité:des substances odorantes-(p. 260). Il est encore plus importantrd'examiner comment, et surtout sous quelle épaisseur minimum, elles modifient.l'attraction au contact, parrexemplecelledes. solides pour l'eau, .c'est-à dire la mouillatbilité. Nous avons ici un moyen d'aborder'la grande question du contact, des attractions. de contact et de leur variation: avee la distance, c'est.à,dire. encore du champ d'attraction. moléculaire (voir plus haut, $p, 259$ ).

d) Mouillabilité: [Devaux,$\left({ }^{\left({ }^{9}\right)}\left({ }^{65}\right)\left({ }^{\left({ }^{6}\right)}\right)\right] .-$ - Pour apprécier la. mouillabilité, on peut tout simplement tremper le corps :lans.l'eau; puis l'en retirer : s'il se mouille-bien, il reste couvert. d'une pellicule aqueuse:persistante (mouillage parfait); sinon la pellicule se rétracte (mouil lage imparfait:ounul). On peut aussi déposer une goutte isolée ou bien effectuer une buéé, c'est-à-dire provoquer la formation de nombreuses:gouttelettes microscopiques: Grandes. ou petites; les gouttes: d'aau s'aplatissent d'autant plus que' le corps est plus: mouillable et peuvent confluer àıla fim; et. inversement; elles: restent d'autant plus convexes et isolées. que le corps est moins mouillable.

J'ai employé ces deux méthodes; et diautres aussi : toutes m'ont. donné des résultats concordants, en expérimentant spécialement sur. le verre. Jo nettoie celui ei par simple flambage, en flamme très: chaude, ce qui détruit instantanément la.pellicule de souillures organiques.qui existent habituellement.

On reconnaìt que l'opération a été bien effectuée à ce fait que la surface du verre ainsi flambée se mouille très bien: Sur une telle surface je déposealors; à l'aide diune solution titrée, une couche mince de oire. Aussitôt, la surface ainsi garnie a perdu sa mouillabilité. Or. l'action se conserve aussi intense seus l'épaaisseur. d'une simple molécule que sous des. épaisseurs'plus grandes. C'estla.preuve directe que le rayon d'attraction de contact.est inférieur au diamètre d'une molécule, c'est-à-dire ici à $1,1.10^{-i} \mathrm{~cm}$ : nous avons cité, cidessus, une valeur moindre encore (p. 259).

J'ai même recommu' qu'une couche monomoléculaire discontinue de cire ou d'huile suffit pour rendre le verre immouillable: Uno couche pesant un tiers'(huile) ounn cinquième (cire) d'une assise simple de molécules suffit pour assurer la non mouillabilité.

L'eau n'arrive pas-à chasser. les.pellicules*monomoléculaires-ainsi fixées; même dans le cas de l'huile. Celle-ei a done perdu,complètement sa fluidité quand elle est fixée par une surface solide. Mais il faut, pour:que cette forte adhérence se manifeste, chasser an préalable par la chaleur, appliquée an verso, toute l'eau d'hygroscopicité que pouvait. retenir le verre.

La mouillabilité n'existe: donc que'par le contact direct, c'est une propriété essentiellement localisée dans une assise unique de molécules, l'assise la:plus-superfieiełle des corps. Nous allons' même voir qu'elle est localisée dans une portion seulement de chaque molécule.

e) Les: lamez monomoléculaires hémimouillables: et: la polarité des molécules [Devaux, $\left.\left(^{65}\right)\right]$. - Dès le début de mes recherches sur les lames solides minces formées sur: l'eau, un fait remarquable avait attiré mon attention: c'est: que, d'une manière constante, ces-lames se montraient: nour mouillables; sur leur face supérieure exposée à l'air, très mouillables' au contraire' surfleur face inférieure, au contact de l'eau : elles sont donc hémir mouillables. Le faittast partieulièrement marqué sur'le sulfure de cuivre:

1. Sur une lame' de: verre ayant reçu: queleques gouttes d'une solution de sulfates de cuivre, envoyons $\mathrm{H}^{2} \mathrm{~S}$ pour obtenir une petlicule de CuS au maximum d'épaisseur (10 à 1.ั mu). Cette pellicule a l'apparence d'être: bien sèche à sa surface supérieure, et d'être mouillée de l'autre còté. Pour le vérifier, versons:un peu.d'eau sur un bout : on voit cette 
eau s'engager aussitôt sous la pellicule, au contact du verre, et s'étendre de proche en proche; on peut ainsi laver très facilement la face inférieure d'une pellicule, sans courir le moindre risque de mouiller la face supérieure. Et cette expérience réussit encore avec des pellicules d'épaisseur monomoléculaire $(0,3 \mathrm{~m} \psi)$.

$2^{\circ}$ Dressons maintenant la lame de verre et enfonçons la verticalement dans de l'eau. La pellicule ne s'y enfonce pas, elle abandonne la surface du verre pour celle de l'eau en se pliant à angle droit : elle flotte bientòt toute entière sur l'eau libre : et ceci a lieu aussi, même si elle n'a qu'une seule molécule d'épaisseur.

$3^{\circ}$ Une pellicule de CuS étant formée dans une cuvette, agitons fortement le liquide pour la briser et noyer les fragments ; beaucoup de ceux-ci sont en effet submergés ; mais si l'on ajoute un peu d'eau de Seltz, ils remontent en grand nombre, chacun rattaché à une bulle. Ces bulles se sont produites parce que chacun des fragments étant imparfaitement mouillé, sa surface supérieure avait emporté une mince couche d'air.

$4^{\circ}$ On peut obtenir aussi la submersion en versant un peu dalcool sur une lame neuve; cet alcool grimpe sur la lame parce qu'il la mouille; à la fin, il la disloque et submerge les fragments. Mais cette fois, il n'y a pas d'atmosphères superficielles sur ces fragments ; l'air y est remplacé par une couche d'alcool retenue fortement.

Ces divers faits prouvent surtout que la face supérieure est peu ou point mouillable. En voici un autre qui se rapporte à la mouillabilité de la face inférieure.

$5^{\circ}$ Formons une large pellicule de CuS dans une cuvette, et appliquons sur elle une lame de verre; submergeons celle-ci et retirons-la. En la retournant, nous trouvons que la pellicule de CuS est collée au verre; elle y est collée par sa face supérieure, celle qui était sèche, de sorte que maintenant l'autre face se trouve à découvert. On voit aussitôt que cette face est parfaitement mouillée, elle est entièrement garnie par une pellicule d'eau bien continue. Le fait est rendu plus frappant si on a rendu auparavant la surface du verre immouillable en la paraffinant; dans ce cas, l'eau n'adhère que là où il y a du CuS.

Or, cet essai réussit encore très bien, même avec une lame d'épaisseur monomoléculaire, malgré les craquelures inévitables, très nombreuses alors, qui empêchent la continuité du mouillage d'être aussi complète. Il arrive même une perturbation accidentelle pleine d'intérêt. En de nombreux endroits des bulles aplaties subsistent entre la pellicule et le verre; cela n'empêche pas l'eau de former une couche continue, même au-dessus des bulles, quoiqu'elle ne soit maintenue alors que par la pellicule de CuS de $0,3 \mathrm{~m} \mu$ !

On peut donc affirmer :

$1^{\circ}$ La grande différence de mouillabilité des deux faces d'une même lame de CuS.

$2^{\circ}$ La persistance de cette différence même quand la lame est réduite à une seule assise de molécules.

Nous avons ici la démonstrátion directe, purement expérimentale, que la molécule de sulfure de cuivre est douée de polarité; elle attire fortement l'eau d'un côté, très peu de l'autre. C'est la confirmation objective des vues les plus récentes sur la constitution de la couche superficielle des liquides et spécialement des lames d'huile (Langmuir) ( ${ }^{53}$ ).

Ces faits sont généraux; je les ai observés sur de nombreuses lames minces de substances solides inorganiques ou organiques. J'y reviendrai un peu plus loin.

f) Les couches monomoléculaires et le frottement. - L'étude de la mouillabilité fait connaitre directement l'adhérence des liquides aux solides. Celle du frottement nous permet de faire une étude parallèle plus délicate, celle de l’adhérence des solides aux solides. Et, par le fait mème qu'un frottement est susceptible de mesure, cette étude permet alors d'apprécier les forces d'adhérence.

$1^{\circ}$ Lord Rayleigh. - Ce problème me préoccupa dès l'origine de mes recherches et il se posa de nouveau devant moi à l'occasion d'une publication que je reçus de lord Rayleigh en 1918 sur les propriétés lubrifiantes et autres des lames minces d'huile (ja). En répondant à lord Rayleigh, le 13 avril 1918, je lui écrivis les lignes suivanles : « Ne pensez-vous pas que la cause essentielle qui gêne le glissement des surfaces mouillées d'eau, pourrait être due à ce que le mouillage est toujours imparfait, quels que soient les soins que l'on apporte au nettoyage? » Et j'ajoutais un peu plus loin : "L'épaisseur de la couche grais- 
seuse suffisante pour assurer le glissement est facile à mesurer : il suffit de répandre un volume déterminé d'une solution titrée d'huile dans la benzine. J'y ai songé depuis longtemps sans avoir eu le temps de faire l'essai. »

Lord Rayleigh me fit l'honneur d'une réponse le 5 mai 1918 , dans les termes suivants (traduction):

“ Je suis tout à fait porté à agréer vos vues. J'espère que vous arriverez à réaliser " votre projet de mesurer la quantité d'huile nécessaire pour rendre les surfaces glissantes “ en se servant d'une solution diluée. Mais quand j'ai essayé cette méthode (pour l'eau) il « y a longtemps, je me suis aperçu qu'elle ne réussissait pas.

« Vous semblez avoir de la persévérance et de l'adresse.

( Les problèmes en question sont, je crois, importants et bien dignes de la peine qu'ils ( peuvent exiger pour les résoudre. Moi, je suis vieux ( 75 ans et demi) et mes recherches (" ne sont pas couronnées de succès comme autrefois ).

$2^{\circ}$ Hardy, 1919. - Un très imporlant mémoire de W.-B. Hardy et du lieutenant J.-K. Hardy a été publié en 1919 sur cette question du frottement et des propriétés lubrifiantes de certaines substances $\left(^{56}\right)$. Les auteurs arrivent à conclure que la friction observée entre deux surfaces propres est due à la cohésion directe entre ces surfaces. Elle peut être suffisante avec le verre pour provoquer l'arrachement d'écailles de la substance. Mais il suffit de l'interposition d'une couche, même mince, de certaines substances, dites actives, pour empêcher cette adhérence, même sous une épaisseur " presque certainement de l'ordre de $1 \mathrm{~m} \mu$ ». Ils ne donnent aucune mesure de l'épaisseur minima sufiisante pour que l'effet lubrifiant se manifeste.

$3^{\circ}$ Devaux, $1923\left({ }^{72}\right)$. - C'est une observation faite le 7 janvier 1923 qui m'a décidé à aborder à mon tour cette question par voie expérimentale. En vue d'étudier la mouillabilité du verre, j'avais flambé plusieurs lames. L'une d'elles s'étant salie d'un peu de poussière, je voulus l'essuyer avec un linge. Je rencontrai une résistance inattendue : le linge s'accrochait littéralement au verre. D'autres lames non flambées présentèrent au contraire un glissement plus ou moins parfait, et la lame en question, graissée volontairement avec les doigts devint à son tour glissante.

a) Adhérence par frottement. - Cette observation est des plus faciles à répéter ici même (expérience) : il suffit d'un flambage de une à deux secondes pour donner au verre le pouvoir de happer le linge. On a l'impression d'une véritable adhérence, l'étoffe est retenue; elle ne cède que par à-coups successifs, on entend même souvent un léger grincement. En même temps, le linge abandonne très vite des impuretés sur le verre (on le voit en essayant de laire une buée) et la friction diminue beaucoup, se change en un glissement de plus en plus facile. Cette perturbation se manifeste moins tôt quand on frotte avec du papier Berzélius.

Au reste, je me suis assuré qu'on peut employer une substance quelconque comme frotteur : bois, verre, aluminium, nickel, cuivre, laiton. Toutefois, le frottement présente des valeurs distinctes pour chaque espèce de substance : rude avec les substances précédentes, il l'est beaucoup moins et devient un vrai glissement avec d'autres, telles que le platine, le soufre, le charbon, etc. L'adhérence de frottement parait donc être une propriété spécifique : c'est, en effet, ce qu'ont démontré les mesures prises dans la suite.

3) Epaisseurs lubrifiantes minima. - Pour déterminer l'épaisseur minimum de lubrifiant, j'ai suivi le procédé dont j'avais parlé à Lord Rayleigh en 1918. Par le procédé des solutions titrées j'ai mis sur des lames de verre préalablement flambées des épaisseurs connues de cire ou d'huile, puis j'ai étudié le frottement. J'ai ainsi reconnu que, tant qu'il existe sur le verre une couche graisseuse de plusieurs molécules, il y a glissement. Pour une seule épaisseur moléculaire le glissement est maintenu à peu près intact; au-dessous, c'est-à-dire avec des molécules distantes les unes des autres, l'effet s'affaiblit vite, mais reste perceptible. Les essais ont été entravés ici, à la limite, par l'existence de traces d'impuretés dans le dissolvant.

La limute d'action d'un enduit graisseux est donc la même, sensiblement, soit à l'égard 


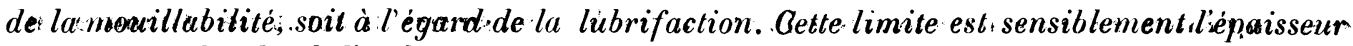
mêne: des:molécules: de!l'enduit.

Y) Glissement et grincement. - Une expérience si mplexpermet de révéler, devant tout un auditaire, à la fois: l'adhérence'entre: surfaces: propress et lo glissement entre: surfaces séparées par une couche monomoléculaire de cire ou d'huile. ll suffit de frotter un bont de beis, tel ‘qu'une allumette; sur du, verre flambé, pour provoquer, un grincement perceptible. Ce grincement, disqaraît quand une couche graisseuse, même monomoléculaire existe. On accentue cerbruit en fixantil'allumette sur un oarton qui joue :le: rôle de résonateur (expériences).

D'autre part, la résistance d'une: lame de luhrifiant, même monomoléculaire, est extraordinaire : si.l'on réserve contre le flambage une portion:diune surface de verre couverte d'une de:ces lames; et،que l'onfrolte avee une baguette de verre; la. surface.cirée est entièrement préservée, tandis que sur tout le:pourtaur elle: est tarrachée et. dép̧olie. La friction opérée peut cependant dépasserr 2.000 . kilogrammes par. centimètre carré.. Nous avons là une manifestation remarquable de l'adhérence des lames monomoléculaires.

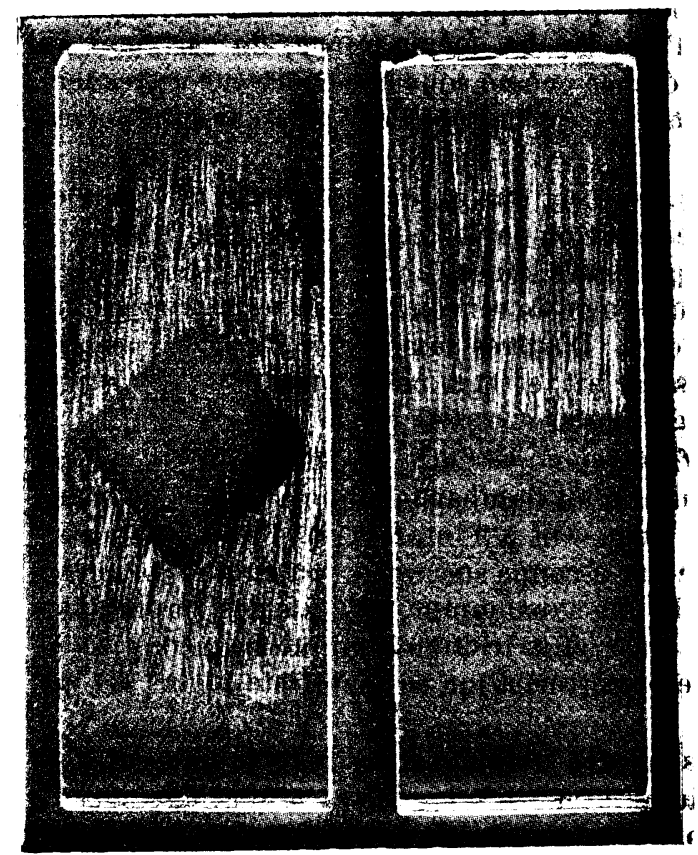

Fig. 19 et. 20w - Par frottement (verre sur. verreł le: verre a été apraché: dans-les:régions: mises à nu (flambées); il a été entièremnt: préservé dans les régions garnies d'une couche cireuse très mince, et préservées du flambage.

Chapitre III.

\section{La structure' des lames monomoleculaires et l'orientation des' molecules.}

a) Historique. - Les faits précédents; et particulièrement' l'existence certaine de lanres monomoléculaires mouillables d'un seul côté, trouvent une: explieation trèsı claire dans la théorie de l'orientation des molécules le long de la surface des: corps et 'spécialement dès liquides: Cette hypothèse a' été nettement formulée par: Hardy, en 19:12-19.13 (Pror. 
Roy. Soc., \$86,'610 631) et peut:être avant (1), et elle a été développée d'une manière indé-

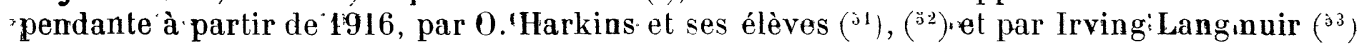
(19f7). 'A cette occasion, dans le beau mémoire qu'il consacre à la question, Il. ILangmuir fait un historique assez détaillé de la découvete des lames monomoléculaires, en analysant les travaux de Lord Rayleigh, de Agnès Pockèl, puis en résumant:mes propres recherches qư'il 'déerit dans ce qu'elles' ont d'essentiel et dont il adopte la conclusion ، " that these films are really one molecule deep $)$ (p. 1862).

IIl part dès tors de cette notion pour recherèher, dans les. affinités 'propres de certains pôles de ehaque mólécule pour.l'eau, une cause d'orientation arrive à démontrer cellezci dans le cas spécíal des acides gras. Chez eeux-ei le groupe CO.OH qui se trouve au bout de 'la chaine constituant la molécule, doít avoir une affinité spéciale pour'lieau, ret.s'appliquer -sur celle-ci, le 'reste de la chàine étant debout, : perpendicubairement à la surface. Il en est bien ainsi car, d'après ses mesures, l'aire occupée par tous les acides grasinormaux de $\mathrm{C}^{14}$ à

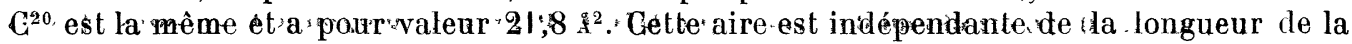
châine, ce qưi s'explique très bien 'dans l'hypothèse de :I'orientation vertiaale detoutes les mołéeules.

Ces recherches ont été étendues ensuite à un très grand nombre de corps gras par idivers autres cherèheurs parmi lesquels' je cfterai'Wøøg' $\left({ }^{67}\right)$, 'N. 4 K. Adam et ses collabora'teurs $\left({ }^{39} 9\right)$, Carrière $\left({ }^{70}\right)$, etc.

'Des' milliers' de mesures ont étét fournies, confirmant toutes, "non seulementl'existence des lames mơomóléculaires, mais l'orientation constante des moléeules, de sorke que.la théorie de l'orientation est 'définitivement démontrée.'On part maintenant de cette'nation pour essayer de déterminer' la forme des moléeutes.

b) Réalisation artificielle de surfac $\_s \mathbf{s}$ lides hémimouillables $\left({ }^{65}\right)$. - L'existencẹ posi' tive : tirée té mes observations, 'de surfaces monomøłéculaires mouilłablesud'un seul côté, 'peut être considérée comme une' autre démonstration, plus directe, que les moléesiles sont doưées de połlarité. Mais cékaım'a.donné l'idée d'essayer de réaliser expérimentalement de 'tèlles-surfaees en provoquant sur une même substance la moullabilité et la non-mouilla'bilifé, et par là, de vérifier que los molécules sont vräiment douées de pôles et qu'on peut les orienter à volonté. Mon idée directrice a été quîl :suffirait pour cela de permettre aux orientations de s'établir au moment de la formation du solide : toute surface solide d'un corps quelconque, où les molécules auront pu. s'orienter librement lors de la solidification, devra présenter une surface mouillable si cette surface s'est solidifiée au contact de l'eau, une surface non mouillablessielless'est sølidifiée en présence de l'air.

Vérification. - Les essais ont vérifié ces prévisions :

$\mathbf{1}^{\circ}$. Des corps; gras, des cires, des lanłe, ont donné ápar refroidissement des plaques de quelques millimètres d'épaisseur. sur lesquelles j'ai constaté une mouillabilité très différente des faces : l'eau roule à da surface

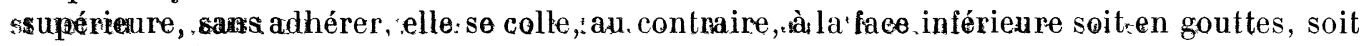
ı mêmeren'pellicule continue.

$: 2^{\circ}$. Pourmes substances solublestdansil'eau (selsinorganiques divers), la démonstration cest déjà̀faite en sens. contraire. Tou tıde.mender en effet ra vu se former des.cristaux flottants sà̀ la : surface edes solutions que l'on concentre. Ils: siunissent souvent même en une croûte

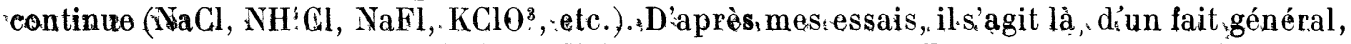
présentant seulement des variation s. IJ'ai pu'm'assurer que:le, flottement est bien dûa au nonmouillage de portions du cristal, et non à des impuretés. Il se maintient même après trans-

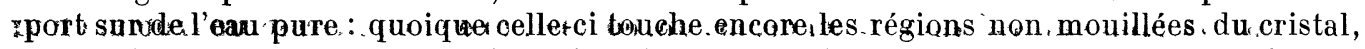
cet qu'elte:les surpłombe, elle: cefuse diy:grimper.: le.cristalse disøout sans,doute, mais uniaquementipar dessous. Oni peut constaten enfin que, sur les.croùtes salines, des, gouttes projetées ne s'étendent pas.

:Les deux.conséquences prévues ont donc été vérifiées.

(1) Plusieurs physiciens ont été condui's à admettre : une orientation desı molécules ‘à ala ‘surface des

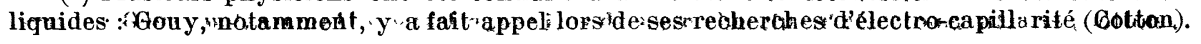


. c) La polaritè des atomes. - On peut mème aller plus loin et se demander si la mouillabilité, c'est-à dire l'attraction pour l'eau, est une propriété moléculaire, ou bien si elle ne serait pas plutôt une propriété atomique. Il suffit pour le vérifier d'essayer d'oblenir des lames hémimouillables avec des molécules monoatomiques, telles que celles des métaux.

Les essais effectués sur le cuivre et sur l'argent ont parfaitement réussi $\left[\operatorname{Devaux}\left({ }^{i 3}\right)\right.$ ].

Une solution d'azotate d'argent, additionnée d'un peu de formol et soigneusement neltoyée à sa surface, est exposée à des vapeurs ammoniacales très diluées. Elle se recouvre très vite d'une couche d'argent métallique, mince d'abord, puis épaisse et brillante. La lame ainsi produite flotte sur la liqueur malgré la grande densité de l'argent $\left({ }^{10}\right),\left({ }^{33}\right)$.

Je me suis assuré, par diverses expériences que la face libre ne se mouille que très difficilement. Si on arrive néanmoins à la submerger, et qu'on la rapproche de la surface, elle se démouille vivement et se remet à flotter. Cette lame d'argent a donc une surface nettement non mouillable.

Or, la face opposée est au contraire très mouillable car si, moyennant certaines précaulions, on recueille la lame d'argent sur une lame de verre cirée, en exposant cette face à l'air, elle se montre parfaitement mouillée et garde une pellicule d'eau continue. L'effet est d'autant plus net qu'à côté le verre ne se mouille pas à cause de la cire qui le garnit.

11 paraît, dès lors, bien évident que c'est l'atome lui-même qui est doué d'attractions polarisées. Nous rejoignons ici, par une voie purement physique, les conceptions de la chimie et spécialement celles de la valence. Une lame mince monomoléculaire d'une substance posée sur l'eau est attirée par celle-ci par des pôles atomiques, absolument comme s'il n'y avait qu'un seul atome de substance et une seule molécule d'eav. Une lame monomo. léculaire est une sorte d'immense molécule plate n'ayant sur chaque face qu'un seul type d'affinité.

On devine, sans qu'il soit besoin d'insister, combien la réalisation expérimentale de telles lames, qui est si facile, va ouvrir de nouvelles voies aux chercheurs. C'est une méthode nouvelle d'étude de la matière à placer sur le même rang que l'étudè des cristaux par les rayons $\mathrm{X}$. On s'en sert déjà de plus en plus pour l'étude de la forme des molécules, mais on ne peut tarder à l'employer pour les études de la dynamique moléculaire, en particulier pour la théorie des actions catalytiques $\left({ }^{1}\right)$.

\section{Chapitre IV.}

\section{Les lames minces et membranes polarisées chez les êtres vivants.}

Pour compléter tous ces aperçus que nous donnent les lames monomoléculaires, je devrais maintenant parler des lames d'albumine et de la constitution moléculaire des cellules vivantes. Je suis obligé de n'en donner qu'un très bref résumé.

a) Les lames très minces d'albumine. - Je les ai découvertes en 1903, et même ma première publication sur les lames minces se rapporte à l'albumine $\left({ }^{23}\right)$. J'avais reconnu dès cette époque, non sans surprise $\left(^{2}\right)$ qu'une goutte d'albumine déposée sur l'eau s'étend à la manière de l'huile, prend des dimensions finales de même ordre de grandeur, et qu'ellesubit en même temps une véritable coagulation, car elle devient une lame manifestement rigide. L'albumine est donc susceptible de s'organiser en membrane sous l'action des forces superficielles. Ce fait m'a, dès le début, beaucoup frappé ; j'y ai vu l'origine et la clef de l'organisation des êtres vivants.

b) Les lames plasmiques. - J'ai constaté, en effet, que si l'on blesse un animal ou une plante, et qu'on mette ce qui s'échappe de la blessure au contact de l'eau, il se produit également une lame d'albumine coagulée, de sorte que j'ai pu intiluler ma note : Sur une réac-

(1) J'ai indiqué un moyen possible d'obtenir des surfaces catalytiques dans le mémoire publié par E. Aubel et Génevois dans le Mémorial des sciences physiques, fascicule VII, sous le titre : L'Etat actuel de la question des ferments. (Gauthiers-Villars, 1929, p. 37).

(2) En réalité, c'est ma mère vénérée qui m'a suggéré cette expérience à laquelle je ne songeais pas. 
tion noucelle et générale des tissus vivants, et j'ajoutai un sous-titre significatif : Essai de. détermination directe des dimensions de la micelle albuminoüde.

c) Les particuir's vivantes élémentaires. - Je pensais bien avoir atteint, en effet, les particules élémentaires de la vie, les fameuses micelles de Nägeli. Car des mesures directes (à l'aide de solutions titrées d'albumine) me donnaient des épaisseurs très voisines de la valeur théorique calculée par Nägeli $(2$ à $3 \mathrm{~m} \mu)$. C'est peu après que je reconnus, sur diverses substances réduites en lames très minces, que les particules solides ou liquides élémentaires de ces substances n'étaient pas des agrégats de molécules, mais les molécults elles-mêmes et que l'albumine aussi donnait des lames monomoléculaires.

Les particules élémentaires de la vie ne sont donc pas des micelles, ce sont des molécules.

d) La structure moléculaire de la cellule. - J'étais ainsi amené logiquement, dès l'année 1903, à aborder le plus passionnant de tous les problèmes, celui de la structure moléculaire des êtres vivants, de l'arrangement de leurs particules élémentaires dans le protoplasma, dans le noyau, dans les membranes, etc. Cependant, j'ai laissé passer de nombreuses années, accumulant des observations sans rien publier à ce sujet, sauf çà et là des notes sur l'adsorption des ions (série de notes de 1896 à 1930, indiquées dans la publication $\left({ }^{86}\right)$ ou sur la mouillabilité des surfaces vivantes $\left({ }^{71}\right)$.

Au cours de cette longue période, de noureaux chercheurs se mirent à l'étude de cetle question, et je dois ici une mention spéciale à Lecomte du Noüy qui, dès 1923, exprima formellement l'opinion qu'une lame monomoléculaire doit exister autour des globules sanguins, et qui ne cesse de fournir depuis de très beaux travaux sur les sérums.

Ce n'est qu'en 1928, vingt-cinq ans après ma première publication, que j'ai publié enfin une note $\left(^{84}\right)$ et un mémoire $\left.{ }^{\left({ }^{86}\right.}\right)$ se rapportant à la structure moléculaire des êtres vivants.

$1^{\circ}$ Structure molêculaire des êtres vivants. - Je remarque tout d'abord que toutes les parties solides des organismes se formant à partir d'éléments fluides et se déposant toujours le long de surfaces limites, toutes leurs particules élémentaires doivent ètre orientées. Elles doivent donc constituer des surfaces à affinités orientées. J'ai pu démontrer qu'il en est réellement ainsi, en rappelant tout d'abord les nombreux cas oû une structure moléculaire, orientée par rapport aux surfaces, est connue depuis longtemps par des propriétés optiques ou mécaniques (membranes, grains d'amidon, etc.). J'avais établi en 1926, que ces mèmes membranes sont aussi hémimouillables $\left({ }^{71}\right)$ et que cette hémimouillabilité cesse au moment de la mort. Or, l'étude des limites du protoplasma avait montré aux cytologistes et aux physiologistes que ces limites sont constamment occupées par une membrane et j'avais montré dès $1904\left({ }^{27}\right)$ que cette membrane plasmique était produite par coagulation, absolument comme les membranes d'albumine. De cet ensemble de faits, j'ai pu conclure que ces membranes plasmiques, premières manifestations visables d'une organisation, ont nécessairement leurs molécules orientées; chaque unité vivante de la cellule, protoplasma, noyaux, plastes ou mitochondries, nous apparaît comme limitée par une véritable lame mince solide en forme de sac clos, dans laquelle toutes les molécules sont polarisées, toutes les affinités réparties en deux champs d'action : l'un externe, l'autre interne. Ce sont des surfaces closes limitées, hémimoléculaires, dont le type est général chez tous les êtres vivants et ne se retrouve nulle part ailleurs dans tout l'Univers.

$2^{\circ}$ Activité des membranes vivantes. - Or, cette organisation remarquable est liée à la formation et à l'entretien de ces membranes; j'ai pu le montrer en $1930\left({ }^{90}\right),\left({ }^{91}\right)$, par une étude spéciale des affinités dont la cellule est le théâtre. Les affinités libres sont, en effet, tout à fait absentes dans la masse du protoplasma, du noyau, etc. Dès lors, les activités vitales ne pouvant exister dans les masses plasmiques doivent être nécessairement localisées le long des surfaces plasmiques, c'est-à-dire des membranes vivantes. Des faits positifs, en particulier ceux observés par Warburg et par Génevois, sont conformes à cette manière de voir.

La moindre cellule vivante nous apparait ainsi comme un laboratoire merveilleux, un laboratoire moléculaire qui ne travaille que sur les molécules individuelles, mettant à profit 
toutes'les propriéfés de ces molécules :, nature, dimensions, formes, pòles attractîs, aussi bien que leurs mouvements, pour'se construire et se renouveler lui-méme. Au point xle vue cosmique, 'toute cellule-vivante se'montre comme un centre organisateur des forces et des mouvements moféculaires, un constructeur catalytique de molécules·spéeifiques, spéeifiquement arrangées en un'tout supérieur.

Chaque cellule, chaque individu vivant, est un monde qui se crée et se recrée-sans cesse, semblable à lui-mième, par ses propres molécules spécifiques, et c'est dans la constitution intime de ces molécules vivantes que subsiste, reculé mais toujours insondable, le grand mystère de' la vie.

\section{$* *$}

Conclusion. - J'arrête.ici.cesibrefs aperçus que nous donne l'étude des lames monomoléculaires.

Qu'on les examinedans. le monde inanimé ou dans les êtres vivants, ces lames nous montrent.un ensemble.merveilleux et insoupconné encore il y a quelques années.

La mise en:surface se révèle comme une mise en action d'énergies qui arrangent partout les molécules et les forces moléculaires, avec une précision et une ampleur qui nous rempliraient: d'étonnement si nous pouvions.les. distinguer. L'aşpect d'un millimètre carré d'une lame monomoléculaire.de cire, avec. ses 1000 milliards de molécules, mieux rangées que lą̧ plus splendide mosaïque, ou.celui d'un fragment.de membrane nucléop̉lasmique dans une cellule végétale ou animale, avec son activité prodigieuse, nous saisirait d'admiration.

“Qui.a créé ees choses et qui diṣpose en ordre leur armée?... » pourrions-nous dire en paraphrasant un Prophète qui parlait.des étoiles (Esaïe, 40, 26). Mais une tèlle question dépasse ce que peut dire la Science humaine. Le moindre atome de matière, comme la moindre cellule vivante est et restera ، toujours un mystère; nous devons nous prosterner devant la souveraine.Puissance qui a créé les mondes, les molécules et les êtres vivants. 


\section{BIBLIOGRAPHIE}

(1) Jamir (J.). Ann. de physique et de chimie, 29 (1850) et 31 (1851).

(2) Van der Mersbrugghe Mém. divers dans le Bull. de l'Académie des sciences de Belgique, à partir de 1870.

(3) Reinold et Rucker. Phil. Trans., 174, II (1883), p. 645; 184 A (1893), p. 505.

(4) E. Јоноnnotт. Phil. Mag., (5) 47 (1899), p. 501.

(5) Amiral Coové Le filage de l'huile, C. R., 104 (1887), p. 586.

$\left(^{(}\right)$H. Devaux. Mouvements spontanés de certains corps à la surface de quelques liquides, La Nature, 16 e année, 777 (1888), p. 331.

$\left({ }^{7}\right)$ LoRd Rayleigh. Measurements of the Amount of oil nécessary in order to check the motions of camphor upon water, Proc. Royal Soc. Lındon, 47 (1890), p 364.

$\left.{ }^{8}\right)$ Lord Rayleigh. On the superficial viscosity of water, Id., 48 (1890), p. 127.

(9) Rontgro. Wiedemann's Ann, t. 41 (1890), p. 320

(10) Lord Rayleigh. Phil. Mag., 30, 5e série (1890), p. 285.

(11) Agrès Pockel. Lettre à Lord Rayleigh dans Nature, 43 (1890), p. 437.

(12) Lond Rayleigr. On the tension of recently formed liquid surfaces, Proceed. roy. Soc., 48 (1890), p. 244.

(13) Lord Rayleigh. Experiments upon surfaces-films, Phil. Mag., כ̌e série, 33 (1892), p. 366.

(14) Agrès Pogkel. Nature, 46 (sept. 1892). (Anomalies de la tension superficielle par des couches minces.)

(15) Agnès Pogrel. Nature, 48 (sept. 1893). (ld.)

(16) Agnès Pockel. Nature, 50 (1894), p. 223. (Extension des huiles.)

(17) K.-T. Fischer. Die geringste Dicke von Flussigkeitshaütchen, Inaug. Diss, juin 1896 (Munich 1897).

(18) Agnès Pockel. Ann. der Phys. und Chemie, 67 (1899), p. 668.

(19) Lord Rayleigh. Investigations in capillarity, Phil. Mag., 48 (1899), p. 337.

("i) Vircknt. Epaisseur des couches de passage, Ann. de Chimie et de Physique, 7e série, 19 (1900), p. 421.

(21) Quircke. Nombreux mémoires de 1868 à 1904 (voir Traité de physique de Chwolson, I, 3, 639).

(28) A. Pockels. Wied. Ann., 8 (1902), p. 8 ว̆4.

(23) Lord Rayleigh. Drops and surface Tension, Roy. Inst. of gr. Britain, 3 april 1903.

(24) Ramsden. Proc. Roy. So:., 72 (1903, p. 256.

$\left({ }^{25}\right)$ H. Devaux. Sur une réaction nouvelle et générale des tissus vivants : Essai de détermination directe des dimensions de la micelle albuminoïde. (Proc. verb. Soc. des Sc. phys. et nat., Bordeaux, nov. 1903.)

( ${ }^{86)}$ H. Devaux. Recherches sur les lames très minces, liquides ou solides. (Proc. verb. Soc. des Sc. phys. et nat., Bordeaux, 3 déc. 1903.)

(27) H. Drvaux. Membranes de coagulation par simple contact de l'albumine avec l'eau. (Proc. verb. Soc. des Sc. phys. et nat. Bordeaux, janvier 1904.)

$\left.{ }^{\left({ }^{3} 3\right.}\right)$ H. Devaux. Sur l'épaisseur critique des liquides et des solides réduits en lames très minces. $(C . R$. Soc. française de phy -ique, 18 mars 1904.)

('9) H. Devaux. Comparaison de l'épaisseur critique des lames très minces avec le diamètre théorique des molécules. (Proc, verb. Soc. des Sc. phys. et nat., Bordeaux, 14 avril 1904.)

$\left.{ }^{(30}\right)$ H. Devaux. Sur l'épaisseur critique des solides et des liquides réduits en lames très minces, J. phys. (4), 3 (1904), p. 450.

(31) W.-W. Metcalf. Uber feste Peptonhaütchen auf einer Wasserfläche und die Ursache ibrer Entstehung, Z. für phys. Chem., 52 (1905), 1, Leipzig.

(32) J. Konigsberger und J.-W Muller. Physik. Z., t. 6 (1905), 847 et 12 (1911), p. 606.

( ${ }^{33}$ ) Shorter (S -A.). Phil. Mag., 62, fév. 1906, p. 317. (Surf. des sol. de saponine.)

(34) Jononmott (E -S.). Phil. Mag., 62 (1906), 776 (lame noire).

(35) Herbert Starspield. Proc. Roy. soc., vol. 77 (1906), A, 314 (lames noires).

(36) J. Perrin. La grandeur des molécules, $C . R, 147$ (1908), p. 594 et nombreux autres notes ou mémoires ultérieurs (voir notice scientifique).

(37) H. Devaux. Lames d'huile étendues sur l'eau : minimum et maximum d'épaisseur d'une lame avec ou sans globules, Soc. fr. de physique, 19 avril 1912.

(38) H. Devaux, Recherches sur les lames d'huile étendues sur l'eau, J. Phys., avril 1912.

$\left.{ }^{39}\right)$ H. Devaux. Sur un procédé de fixation des figures d'évolution de l'huile sur l'eau et sur le mercure, J. Phys. (1912).

(40) H. Devaux. Les lames d'huile étendues sur l'eau et sur le mercure, Revue gén. des sciences, $24^{\mathrm{e}}$ année, 28 fév. 1913, $\mathbf{n}^{\circ}$ 4, p. 143. (Paru en anglais dans Anıual Report of Smithsonian Institution, 1913, 261.)

(41) W.-B. Hardy. The tension of composite fluid surfaces and the mechanical stability of films of fluids, Proc. Roy. soc., 86 (1912), p. 610.

${ }^{(42)}$ Marcelin. Epaisseur des couches très minces à la surface de l'eau, Diplône d'études sup., Paris (1913) Ann. de Phys. (1913).

(43) Agnès Pockel. Physik Z., 15 Jahrgang (1914), p. 39-46.

(44) H. Labrouste. Visibilité de traces de substances étrangères déposées sur une surface d'eau pure, $C . R$, t. 157,7 juillet 1913, p. 44 .

(45) H. Labroustr. Couches monomoléculaires, etc., Bull. soc. fr. de physique, 16 janvier 1914.

(46) H. Labroustr. Transformation moléculuire des couches étendues, C. R., t. 158, 2 mars 1914, p. 627. 
(47) H. Devaux. Sur l'apparition et la disparition des globules dans une mince couche d'huile étendue sur l'eau, Bull. soc. fr. de physique, 20 mars 1914.

(48) H. Devaux. Sur la phase d'équilibre des forces de rassemblement et des forces d'extension agissant sur l'huile déposée sur l'eau; généralisation aux autres substances extensibles (expériences), Bull. soc. fr. de physique, $1^{\mathrm{er}}$ mai 1914

(49) J. Guror. Effet Volta et couches monomoléculaires, $C . R$., t. 157, 27 juillet 1914, p. 311.

(50) H. Devaux. Sur la présence d’un enduit antimouillant à la surface des particules du sable et de la terre végétale, $C . R .$, t. 162,31 janvier 1916, p. 197.

(51) W.-D. Harkins, Brown and E.-G-H. Davies. Structure of the Surfaces of liquids and sol. etc., J. Amer. chem. soc., 39 (1917).

(52) W.-D. Harkiss, E.-G.-H. Davirs and G-L. Chark. The orientation of molecules in the Surfaces of liquides, etc , The Journal of Amer. soc., 39 (1917), 541.

(53) Langmur. The constitution and fundamental properties of solids and liquids. II. Liquids, J. Amer. chem. soc., 38 (1916), p. $221 ; 39$ (1917), p. 1848 (voir p. 1858 pour Oil Films on water).

(54) J Perrin. La stratification des lames liquides, Ann. de phys., t. 10 (1918), p. 160.

(55) Lord Rayleigh. On the lubricating and other Properties of thin oil films, Phil. Mag., 35 (1918), p. 157. Dans ce mémoire l'auteur emploie, p. 14-15, le terme de couche monomoléculaire.)

(56) W.-B. Hardy and lieut. J.-K. Hardy. On the statif Friction and on the lubricating Properties of certains chemical substances, Phil. Mag., 6e série, 38 (1919), p. 32 à 48.

(57) H. Labrouste. Transformations moléculaires dans les couches minces à la surface de l'eau. Thèse, Paris 1920, Ann. de Phys., 9, 14 (1920), p. 164.

(58) H. Devadx. La perméabilité des lames minces. Etude de l'influence des vapeurs et des huiles sur les lames minces solides et liquides, Soc. fr. de physique, 20 mai 1921.

(59) N.-K. Adam. The molecular structure of thin films of palmitic acid on water, Proc. of the roy. Soc., 699 (1921), 99. Nombreux mémoires ultérieurs en 1922, 1923, 1926, 1928.

${ }^{(60)}$ André Marcelin. Tension superficielle des couches monomoléculaires, $C . R .$, t. 173 (1921), p. 38 Extension superficielle des corps solubles ou volatils, $C$. R., t. 173 (1921), p. 79. Mesure de la pression des fluides superficiels, C. R., 176 (1923), p. 502 et autres notes en 1924 et 1925.

(61) Paul Woog. Sur les dimensions des molécules des huiles grasses, etc., $C$. R. (16 août 1921), p 387. Voir aussi $C . R .(16$ janvier 1922), p. 162.

(i2) H. Labrouste. Les couches minces à la surface de l'eau, Soc. fr. de physique, 18 nov. 1921.

(63) Leconte du Noür. Sur l'équilibre superficiel du sérum et de quelques solutions colloïdales, $C . R$., 174 (1922), p 962. Très nombreux mémoires ultérieurs, on en trouvera la liste jusqu'à 1926 dans son livre : Surface équilibria of biological and organic collö̈ds. Ouvrage de la chemical catalog company (1926) Paru en français en 1929 dans les Monographies de l'Institut Pasteur.

(64) M1le Coller. Couches minces de mélanges binaires, Soc. fr. de physique, section de Strasbourg, 10 fév. 1922

(65) H. Devaux. Sur la mouillabilité des surfaces solides et sur l'orientation des molécules superficielles, Soc. $r$ r. de physique, 16 fév. 1923.

(i6) H. Devaux. Ce qu'il suffit d'une souillure pour altérer la mouillabilité d'une surface. Etude du contact d'un liquide avec un solide, J. Phys., t. 4, série 6 (1923), p. 293.

(67) PAUL Woog. Résistance à la rupture, compression latérale et équilibre des couches monomoléculaires de divers corps étendus sur l'eau, $C$. R., t. 176 (1923), p. 1107.

$\left.{ }^{68}\right)$ J.-J. HAAk. Recherches expérimentales sur l'adsorption de l'air par le mercure et la différence de propriétés optiques du mercure liquide et solide, Archives néerlandaises, 3 à 6 (1923), 198.

${ }^{(69)}$ G.-A. Rerser. Mesure des constantes optiques vraies du mercure et étude de la formation des couches superficielles, $I d$. (1923).

(70) J.-F. Carrière. Sur la façon dont l'huile et l'acide oléique se comportent vis-à-vis de l'eau. Recueil des travaux chim. des Pays-Bas (4), 5e série, 15 avril 1924, p. 293, no 4.

(71) H. Devadx. L'injection des lacunes, signe de la mort chez les plantes aquatiques, Revue générale de Botanique (1926), p. 99

(i2) H. Devaux. Le frottement des solides : épaisseur minimum d'un enduit lubrifiant, Soc. fr. de physique, 6 juin 1924 .

(73) H. Devatux. Sur la mouillabilité des métaux et la polarité des atomes, Soc. fr. de physique, 19 juin $19 \overline{2} 5$.

(74) A. Marcelin. Les solutions superficielles, fluides à deux dimensions, Ann. de phys., $10^{\circ}$ série, 55 (nov.-déc. 1925), p. 459.

(75) J.-J. Trillat. Sur l'orientation moléculaire des acides gras. C. R., t. 180 (26 janvier 1925), p. 280 et (4 mai 1925), p. 1329.

(76) L. pв Broglie et J.-J. Trillat. Sur l'interprétation des spectres $\mathrm{X}$ d'acides gras, $C . R$, t. 180 (18 mai 1925), p. 1485 .

(i7) H. Drvadx. Sur la mouillabilité des métaux et la polarité des atomes, Soc. fr. de physique, 5 juin 1925 .

(78) R. Delaplage. Extension de la loi de Gay-Lussac aux solutions superficielles, C. R., 180 (192ら), p, 2020 .

(79) R.-S. Burdon. The Spreading of one liquid on the surface of another, Proceedings Phys Soc., 38 (1926), p. 148. Transactions of Faraday soc., 23 (1927). 
${ }^{(80)}$ J.-J. Trillat. Rayons X et composés organiques à longue chaîne. Recherches spectrographiques sur leurs structures et leurs orientations, Thèse, Paris (1926).

(81) A. Marcelin Les solutions superficielles ou les fluides deux dimensions, La Nature, 2761 (1วั mai 192\%), p. 443 .

(82) H. Devaux. La détermination des poids moléculaires de l'acide abiétique au moyen des lames minces, Soc. des Sc. phys. nat. de Bordeaux (1926-1927).

(83) H. Devaux. Etude des propriétés de la surface du mercure, Id.

(84) H. Devaux. La structure moléculaire de la cellule et des tissus vivants, Soc. fr. de physique, 258 (17 fév. 1928), 345 .

(85) H. Devaux. Lames minces d'épaisseur monomoléculaire sur le mercure, etc., Id. (375)

${ }^{86}$ ) H. Devadx. La structure moléculaire de la cellule végétale, Bull. Soc. bot. de France, 75, 4 (1928), p. 88 (avec bıbliographie de mes recherches de physique moléculaire).

(87) Revé Delaplace. Solutions superficielles; lois de Mariotte et de Gay-Lussac, J. Phys., t. 9(1928), p. 111.

(88) Fahir Emir. Epaisseur d'un film d'acide oléique, C. R., 188 (1929), p. 1667. - Solutions superficielles et vernis moléculaires, etc $C . R ., 189$ (1929), p. 239. - Solutions superficielles sur le mercure, etc. C. R., 190 (1930), p. 176. - Couches monomoléculaires sur le mercure, Soc. fr. de physique (1930), p. 185, $\mathrm{n}^{\circ} 287$.

${ }^{(89)}$ H. Devalux. Départ et rétention des molécules du camphre et d'autres substances odorantes, Soc. fr. de phy ique (1930), 523, $\mathrm{n}^{\circ} 290$.

(4ii) H. Devaux. Les affinités cellulaires, Bull. Soc. bot. de France, 77 (1930), p. 144 à 159.

(91) H. Devaux. Le lien entre l'organisation et l'activité vitale; Rôle des membranes plasmiques, $C . R$., $190(1930)$ p 1241

(92) J.-J. Trillat. Structure interne et superficielle des liquides organiques à longue chaîne, Soc. de phys., n० $292(1930)$, p. 715.

(93) G. Bouнzт. Polarisation elliptique par réflexion à la surface des liquides, etc. Thèse, Paris (1930).

(94) J. Guastalla. C. R., 189 (1929), p. 241.

(95) H. Devaux et E. Auber. L'adsorption des ions par le verre, Proc. verb. Soc. Se. phys. et nat., Bordeaux, (192,)-1926). 


\section{TABLE DES MATIERES}

\section{CHAPITRE I}

\section{Historique des lames monomoléculaires.}

1. Introduction, 238.

2. L'extension de l'huile et des vapeurs sur l'eau et sur le mercure et les actions mécaniques qu'elles peuvent produire, 23 ?

3. La découverte d'une épaisseur critique pour les lames d'huile, 241. - a) Lord Rayleigh (1890), 241. - b) Röntgen, 241. - c) L. Rayleigh et Agnès Pockel (1890-1899), 242. - $d$ ) Fischer (1899), 242. - e) Devaux (1903-1912): 242. - 1. Limitation de l'extension de l'huile, 244: 2. Apparition de la cohésion par resserrement, 245. - 3. Mesures d'épaisseurs, 245. - $f$ ) Résumé, 246.

4. Les lames minces solides, 247. - a) Lord Rayleigh, Agnès Pockel, 247. - b) Devaux, 247. -c) Cohésion et épaisseur, 247.

5. La découverte des lames monomoléculaires, 248. - a) Lord Rayleigh (1890-1903), 248. - b) Devaux (1903) : 1. Premier tableau d'épaisseur théorique et expérimentale, 249. — 2. Démonstration publique et tableaux, 250. - c) Remarque, 252. - d) Lames sur mercure, 253. - e) Conclusion, 254 .

\section{GHAPITRE II}

\section{Les propriétés physiques des lames monomoléculaires, 255.}

1. Propriétés optiques, 256.

2. Stabilité des lames minces, instabilité des lames épaisses, 256.

3. Propriétés mécaniques, 257. - a) Cohésion, 258. - b) Expansibilité, 258. - c) Fluidité, 258. - d) Equilibre entre lame et globules, 258. - e) Rigidité, 259. - $f$ ) Perméabilité, 259.

4. Rapports de la lame avec son support, 260. - a) Adhérence, 260. - b) Adsorption, 260. - c) Les souillures monomoléculaires, 260. - d) Mouillabilité, 261. - e) Les lames monomoléculaires hémimouillable et la polarité des molécules, 261. - f) Les couches monomoléculaires et le frottement. 261.-1) Lord Rayleigh, 261. - 2) Hardy, 262. - 3) Devaux : $\alpha$. Adhérence par frottement, 262. - $\beta$. Epaisseur lubréfiant, 263. $-\gamma$. Glissement et grincement, 264.

\section{GHAPITRE III}

La structure des lames monomoléculaires et l'orientation des molêcules, 264. - a) Historique, 265. - b) Réalisation artificielle de surfaces solides hémimouillables, 265 . - c) La polarité des atomes, 266.

\section{CHAPITRE' IV}

Les lames minces et membranes polarisées chez les êtres vivants, 266 . - a) Les lames très minces d'albumine, 266. - b) Les lames plasmiques, 266. - c) Les particules vitales élémentaires, 267 . - $d$ ) La structure moléculaire de la cellule, 267. - 1. Structure moléculaire, 267. - 2. Activité des membranes, 267. - e) Conclusion, 268. - Bibliographie, 269. 